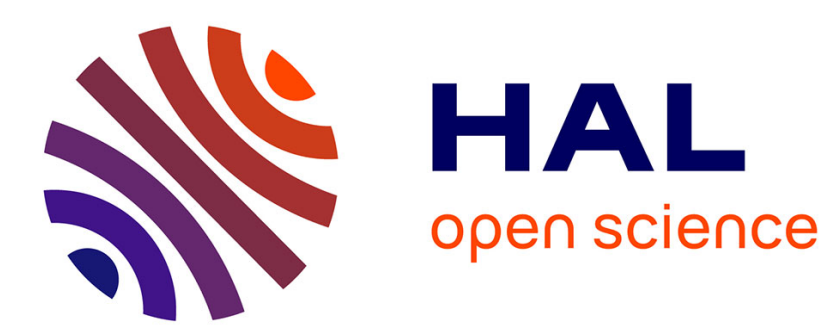

\title{
On the monofractality of many stationary continuous Gaussian fields
}

\author{
Antoine Ayache
}

\section{To cite this version:}

Antoine Ayache. On the monofractality of many stationary continuous Gaussian fields. Journal of Functional Analysis, 2021, 281 (7), pp.109111. 10.1016/j.jfa.2021.109111 . hal-03195594

\section{HAL Id: hal-03195594 https://hal.science/hal-03195594}

Submitted on 11 Apr 2021

HAL is a multi-disciplinary open access archive for the deposit and dissemination of scientific research documents, whether they are published or not. The documents may come from teaching and research institutions in France or abroad, or from public or private research centers.
L'archive ouverte pluridisciplinaire HAL, est destinée au dépôt et à la diffusion de documents scientifiques de niveau recherche, publiés ou non, émanant des établissements d'enseignement et de recherche français ou étrangers, des laboratoires publics ou privés. 


\title{
On the monofractality of many stationary continuous Gaussian fields
}

\author{
Antoine Ayache \\ Université de Lille
}

\begin{abstract}
In this article we focus on a general real-valued continuous stationary Gaussian field $X$ characterized by its spectral density $|g|^{2}$, where $g$ is any even realvalued deterministic square integrable function. Our starting point consists in drawing a close connection between critical Besov regularity of the inverse Fourier transform of $g$ and $\alpha_{X}$ the random pointwise Hölder exponent function of $X$, which measures local roughness/smoothness of its sample paths at each point. Then, thanks to Littlewood-Paley methods and Hausdorff-Young inequalities, under weak conditions on $g$, we show that the random function $\alpha_{X}$ is actually a deterministic constant which does not change from point to point. This result means that the field $X$ is of monofractal nature. Also, it is worth mentioning that such a result can easily be extended to the case where $X$ is no longer stationary but has stationary increments.
\end{abstract}

Running Title: Monofractality of stationary Gaussian fields

Key Words: Sample path behavior, Hölder regularity, spectral density, Besov space, Littlewood-Paley decomposition.

AMS Subject Classification: 60G17, 42B35, 42B05.

\section{Introduction}

Roughly speaking, a stochastic field/process is of multifractal nature (see e.g. [11, $17,19,24,15,16])$ when local roughness/smoothness of its (sample) paths, measured 
through pointwise Hölder exponent, widely changes from point to point. It is tempting to believe that such an heterogeneous erratic behavior of paths would be incompatible with a stationarity property in distribution of the field/process or its increments, since the latter property would somehow oblige increments to behave rather homogeneously from point to point. Yet, a long time ago, it was shown by Jaffard in his important article [18] that, in spite of stationarity of their increments, most of the non-Gaussian Lévy processes are of multifractal nature. More recently, in [7] Balança has also obtained such multifractality results in a framework including the linear fractional stable motion. The latter is a very classical moving average stochastic process with heavytailed stable distributions and stationary increments. It is worth mentioning that, in contrast with the Lévy processes studied in [18], paths of the linear fractional stable motion can be continuous functions provided that its Hurst parameter is large enough (see [13, 23]).

The examples of multifractal stochastic processes with stationary increments which have been exhibited in $[18,7]$ are widely non-Gaussian; this brings us naturally to the following question: are there Gaussian examples of such processes/fields? If the assumption on the stationarity of increments is dropped then the answer to the question is positive: using results in $[4,6]$, one can construct Gaussian multifractional Brownian motions with continuous paths having multifractal natures. The primary motivation of our present work is to determine in the framework of stationary continuous Gaussian fields general sufficient conditions under which the answer to the question becomes negative. Roughly speaking, our results show that such a field is often of monofractal nature.

We focus on the centred real-valued stationary Gaussian field $\{X(t)\}_{t \in \mathbb{R}^{N}}$ of the general form:

$$
X(t):=\int_{\mathbb{R}^{N}} e^{-i t \cdot \xi} g(\xi) d \widehat{W}(\xi), \quad \text { for each } t \in \mathbb{R}^{N},
$$

where $t \cdot \xi$ is the usual inner product of the vectors $t$ and $\xi$, and where $g$ is any arbitrary deterministic even ${ }^{1}$, real-valued and non-negative function belonging to $L^{2}\left(\mathbb{R}^{N}\right)$, the Hilbert space of the complex-valued functions on $\mathbb{R}^{N}$ which are square integrable with respect to the Lebesgue measure. Notice that the stochastic integral $\int_{\mathbb{R}^{N}}(\cdot) d \widehat{W}$ (see for instance the Chapter 1 in [3]) is defined on the whole space $L^{2}\left(\mathbb{R}^{N}\right)$ as follows: for

\footnotetext{
${ }^{1}$ That is $g(\xi)=g(-\xi)$, for Lebesgue almost all $\xi \in \mathbb{R}^{N}$.
} 
any arbitrary deterministic function $f \in L^{2}\left(\mathbb{R}^{N}\right)$, one has

$$
\begin{aligned}
& \int_{\mathbb{R}^{N}} f(\xi) d \widehat{W}(\xi):=(2 \pi)^{N / 2} \int_{\mathbb{R}^{N}} \mathcal{F}^{-1}(f)(x) d W(x) \\
& :=(2 \pi)^{N / 2}\left(\int_{\mathbb{R}^{N}} \operatorname{Re}\left(\mathcal{F}^{-1}(f)(x)\right) d W(x)+i \int_{\mathbb{R}^{N}} \operatorname{Im}\left(\mathcal{F}^{-1}(f)(x)\right) d W(x)\right),
\end{aligned}
$$

where $\operatorname{Re}(\cdot)$ and $\operatorname{Im}(\cdot)$ are the real and imaginary parts, $\int_{\mathbb{R}^{N}}(\cdot) d W$ is the classical Wiener stochastic integral, and $\mathcal{F}^{-1}$ is the inverse of the Fourier transform map $\mathcal{F}$ from $L^{2}\left(\mathbb{R}^{N}\right)$ to itself. We mention that $\mathcal{F}(f)$, the Fourier transform of the function $f$, is very often denoted by $\widehat{f}$. We assume it to be defined according to the very usual convention that $\widehat{f}(\xi)=\int_{\mathbb{R}^{N}} e^{-i \xi \cdot x} f(x) d x$, for all $\xi \in \mathbb{R}^{N}$, when $f \in L^{1}\left(\mathbb{R}^{N}\right)$. Therefore, under the same assumption on $f$, the inverse Fourier transform $\mathcal{F}^{-1}(f)$ has to be defined as: $\mathcal{F}^{-1}(f)(x)=(2 \pi)^{-N} \int_{\mathbb{R}^{N}} e^{i x \cdot \xi} f(\xi) d \xi$, for all $x \in \mathbb{R}^{N}$.

Observe that (1.1), (1.2) and an elementary property of the Fourier transform imply that the field $\{X(t)\}_{t \in \mathbb{R}^{N}}$ can be expressed as:

$$
X(t)=(2 \pi)^{N / 2} \int_{\mathbb{R}^{N}} \mathcal{F}^{-1}(g)(x-t) d W(x), \quad \text { for all } t \in \mathbb{R}^{N} .
$$

Also, observe that similarly to the function $g$ the function $\mathcal{F}^{-1}(g)$ is even and realvalued. Thus, it follows from (1.3) that the field $\{X(t)\}_{t \in \mathbb{R}^{N}}$ itself is real-valued.

Furthermore, it is important to emphasize that, due to the isometry property of the classical Wiener integral and to that of the Fourier transform map, the stochastic integral $\int_{\mathbb{R}^{N}}(\cdot) d \widehat{W}$ itself satisfies the following isometry property: for each $f \in L^{2}\left(\mathbb{R}^{N}\right)$, one has

$$
\mathbb{E}\left(\left|\int_{\mathbb{R}^{N}} f(\xi) d \widehat{W}(\xi)\right|^{2}\right)=\int_{\mathbb{R}^{N}}|f(\xi)|^{2} d \xi=(2 \pi)^{N} \int_{\mathbb{R}^{N}}\left|\mathcal{F}^{-1}(f)(x)\right|^{2} d x .
$$

We mention that throughout our article, the same symbol $|\cdot|$ is used to denote the absolute value on $\mathbb{R}$, the modulus on $\mathbb{C}$ (the set of the complex numbers) and the Euclidian norm on $\mathbb{R}^{N}$. Also, we mention that the isometry property in (1.4) can equivalently be expressed as: for all $f_{1} \in L^{2}\left(\mathbb{R}^{N}\right)$ and $f_{2} \in L^{2}\left(\mathbb{R}^{N}\right)$ one has

$$
\begin{aligned}
\mathbb{E}\left(\int_{\mathbb{R}^{N}} f_{1}(\xi) d \widehat{W}(\xi) \times \overline{\int_{\mathbb{R}^{N}} f_{2}(\xi) d \widehat{W}(\xi)}\right) & =\int_{\mathbb{R}^{N}} f_{1}(\xi) \overline{f_{2}(\xi)} d \xi \\
& =(2 \pi)^{N} \int_{\mathbb{R}^{N}} \mathcal{F}^{-1}\left(f_{1}\right)(x) \overline{\mathcal{F}^{-1}\left(f_{2}\right)(x)} d x
\end{aligned}
$$


Then it follows from (1.1) and (1.5) that the covariance function of the centred realvalued stationary Gaussian field $\{X(t)\}_{t \in \mathbb{R}^{N}}$ is given by:

$$
\operatorname{Cov}\left(X\left(t^{1}\right), X\left(t^{2}\right)\right)=\int_{\mathbb{R}^{N}} e^{-i\left(t^{1}-t^{2}\right) \cdot \xi}|g(\xi)|^{2} d \xi, \quad \text { for all }\left(t^{1}, t^{2}\right) \in \mathbb{R}^{N} \times \mathbb{R}^{N} .
$$

Thus, it turns out that $|g|^{2}$ is the spectral density of $\{X(t)\}_{t \in \mathbb{R}^{N}}$. Moreover, the fact that $|g|^{2}$ can be any arbitrary non-negative even function in $L^{1}\left(\mathbb{R}^{N}\right)$ implies that $\{X(t)\}_{t \in \mathbb{R}^{N}}$ can be of the same distribution as any arbitrary centred real-valued stationary Gaussian field having an absolutely continuous spectral measure with respect to the Lebesgue measure (see [12, 28] for instance).

In order to obtain results on path behavior of the field $X=\{X(t)\}_{t \in \mathbb{R}^{N}}$, it is useful to introduce the function $V_{X}$ called the variogram of $X$ and defined, for all $h \in \mathbb{R}^{N}$, as:

$$
V_{X}(h):=\mathbb{E}\left(|X(h)-X(0)|^{2}\right)=\mathbb{E}\left(|X(\tau+h)-X(\tau)|^{2}\right),
$$

where $\tau \in \mathbb{R}^{N}$ is arbitrary and fixed. Observe that it follows from (1.1), (1.6), (1.4) and an elementary property of the Fourier transform that, for each $h \in \mathbb{R}^{N}$, one has

$$
V_{X}(h)=\int_{\mathbb{R}^{N}}\left|\left(e^{-i h \cdot \xi}-1\right) g(\xi)\right|^{2} d \xi=(2 \pi)^{N} \int_{\mathbb{R}^{N}}\left|\mathcal{F}^{-1}(g)(x+h)-\mathcal{F}^{-1}(g)(x)\right|^{2} d x .
$$

Throughout our article, we always assume that $V_{X}$ satisfies ${ }^{2}$ the following condition:

$\left(\mathcal{H}_{0}\right)$ There is a constant positive critical exponent $\bar{s} \in(0,1)^{3}$ such that one has

$$
\sup _{h \in \mathbb{R}^{N}}\left\{|h|^{-2 s} V_{X}(h)\right\}<+\infty, \quad \text { for all } s \in(0, \bar{s}),
$$

and

$$
\sup _{h \in \mathbb{R}^{N}}\left\{|h|^{-2 s} V_{X}(h)\right\}=+\infty, \quad \text { for all } s \in(\bar{s}, 1) .
$$

Remark 1.1 In view of the second equality in (1.7), the critical exponent $\bar{s}$ in the condition $\left(\mathcal{H}_{0}\right)$ can be expressed in terms of Besov regularity of the function $\mathcal{F}^{-1}(g)$ as follows:

$$
\bar{s}=\sup \left\{s \in(0,1): \mathcal{F}^{-1}(g) \in B_{2, \infty}^{s}\left(\mathbb{R}^{N}\right)\right\} .
$$

\footnotetext{
${ }^{2}$ Notice that we always use the convention that $0 / 0=0$.

${ }^{3}$ Notice that $\bar{s}$ is strictly positive and strictly less than 1 .
} 
One knows from e.g. Theorem 6.2.5 on page 144 in [8], that the Besov space $B_{2, \infty}^{s}\left(\mathbb{R}^{N}\right)$ in (1.10), and more generally the Besov space $B_{p, \infty}^{s}\left(\mathbb{R}^{N}\right)$, for any $p \in[1,+\infty]$, can be defined in the following way:

Definition 1.2 Let $p \in[1,+\infty], s \in(0,+\infty)$ and $m \in \mathbb{N} \cap(s,+\infty)$ be arbitrary and fixed. A real-valued (or more generally complex-valued) function $f$ on $\mathbb{R}^{N}$ belongs to the Besov space $B_{p, \infty}^{s}\left(\mathbb{R}^{N}\right)$ if and only if $f$ is in the space $L^{p}\left(\mathbb{R}^{N}\right)$ and satisfies

$$
\sup _{h \in \mathbb{R}^{N}}\left\{|h|^{-s}\left(\int_{\mathbb{R}^{N}}\left|\Delta_{h}^{m} f(x)\right|^{p} d x\right)^{1 / p}\right\}<+\infty,
$$

where, for all $h, x \in \mathbb{R}^{N}, \Delta_{h}^{m} f(x)$ is the $m$-th order increment of $f$ defined as:

$$
\Delta_{h}^{m} f(x):=\sum_{n=0}^{m}\left(\begin{array}{l}
m \\
n
\end{array}\right)(-1)^{n} f(x+n h) .
$$

Remark 1.3 Using (1.6) and (1.8), it can easily be seen that, for any arbitrary fixed $s \in(0, \bar{s})$, there is a finite constant $c_{s}$ such that the inequality

$$
\mathbb{E}\left(\left|X\left(t^{\prime}\right)-X\left(t^{\prime \prime}\right)\right|^{2}\right) \leq c_{s}\left|t^{\prime}-t^{\prime \prime}\right|^{2 s}
$$

holds for all $t^{\prime}, t^{\prime \prime} \in \mathbb{R}^{N}$. Thus it results from a Gaussian classical corollary of a strong version of the well-known Kolmogorov's continuity Theorem (see e.g. Theorems A6 and $\mathrm{A} 5$ in [3]), that the field $X$ has a modification whose paths are with probability 1 Hölder continuous functions of any arbitrary order $s^{\prime} \in(0, \bar{s})$ on each compact subset of $\mathbb{R}^{N}$. Throughout our article $X$ is always identified with this Hölder continuous modification of it.

Even if paths of $X$ satisfy on each compact subset of $\mathbb{R}^{N}$ a global Hölder regularity condition of any arbitrary order $s^{\prime} \in(0, \bar{s})$, their local behavior in neighborhoods of some points of $\mathbb{R}^{N}$ could be more regular than that. The pointwise Hölder exponent function (see e.g. $[2,3,17,24]$ ), defined below, allows to account for such a phenomenon by providing at each point $\tau \in \mathbb{R}^{N}$ an asymptotic estimate for the local oscillations of a path of $X$ around $\tau$.

Definition 1.4 The pointwise Hölder exponent function of an arbitrary continuous path $X(\cdot, \omega)$ of the field $X$ is the function $\alpha_{X}(\cdot, \omega)$ defined, for all $\tau \in \mathbb{R}^{N}$, as:

$$
\alpha_{X}(\tau, \omega):=\sup \left\{\alpha \in[0,1]: \limsup _{r>0, r \rightarrow 0}\left\{r^{-\alpha} \operatorname{Osc}_{X}(\tau, r, \omega)\right\}<+\infty\right\},
$$


where, for any real number $r \in(0,1]$,

$$
\operatorname{Osc}_{X}(\tau, r, \omega):=\sup \left\{\left|X\left(t^{\prime}, \omega\right)-X\left(t^{\prime \prime}, \omega\right)\right|:\left(t^{\prime}, t^{\prime \prime}\right) \in \mathcal{B}(\tau, r)^{2}\right\}
$$

is the oscillation of the path $X(\cdot, \omega)$ on the ball $\mathcal{B}(\tau, r):=\left\{t \in \mathbb{R}^{N}:|t-\tau| \leq r\right\}$.

The singularity spectrum of the path $X(\cdot, \omega)$ is the non-negative function $\rho_{X}(\cdot, \omega)$ whose value $\rho_{X}(\alpha, \omega)$, at each $\alpha \in[0,1]$, is the Hausdorff dimension (see e.g. [14]) of the level set $\left\{\tau \in \mathbb{R}^{N}: \alpha_{X}(\tau, \omega)=\alpha\right\}$. Thus $\rho_{X}(\cdot, \omega)$ provides synthetic geometrical informations on the repartition of the values of the function $\alpha_{X}(\cdot, \omega)$. When the support of $\rho_{X}(\cdot, \omega)$, that is the topological closure of the set $\left\{\alpha \in[0,1]: \rho_{X}(\alpha, \omega) \neq 0\right\}$, reduces to one point (or more generally when it is with empty interior) then the path $X(\cdot, \omega)$ is said to be of monofractal nature, otherwise it is said to be of multifractal nature.

Remark 1.5 Combining the condition $\left(\mathcal{H}_{0}\right)$ with Remark 1.3 and Theorem 1.74 on page 37 in [3], it follows that, for each fixed $\tau \in \mathbb{R}^{N}$, there exists $\widetilde{\Omega}_{\tau}$ an event of probability 1 , which a priori depends on $\tau$, such that, one has

$$
\alpha_{X}(\tau, \omega)=\bar{s}, \quad \text { for every } \omega \in \widetilde{\Omega}_{\tau} .
$$

Question 1.6 Does the equality (1.15), which holds for all $\tau \in \mathbb{R}^{N}$, necessarily imply that with probability 1 any path of the field $X$ is of monofractal nature?

The answer to this question is non-trivial. Indeed, a result very similar to (1.15) holds for the pointwise Hölder exponent of the linear fractional stable motion (see for instance [5]); however, as we have already mentioned, it has been shown in [7] that the latter stochastic process is of multifractal nature.

In fact, the weakness of the result (1.15) is that one only knows that it is valid on an event of probabilty 1 , namely $\widetilde{\Omega}_{\tau}$, which depends on $\tau$. Therefore, it seems natural to wonder whether there exists an event of probability 1 not depending $\tau$, denoted by $\Omega^{*}$ for instance, such that one has

$$
\alpha_{X}(\tau, \omega)=\bar{s}, \quad \text { for all }(\tau, \omega) \in \mathbb{R}^{N} \times \Omega^{*} .
$$

If one manages to show that (1.16) holds, then a straightforward consequence will be that on the event $\Omega^{*}$ of probability 1 any path of the field $X$ is of monofractal nature. 


\section{Statements of the main results}

We already know from Remark 1.3 that (1.16) is valid when the equality $\alpha_{X}(\tau, \omega)=\bar{s}$ in it is replaced by the inequality $\alpha_{X}(\tau, \omega) \geq \bar{s}$. Thus, one only has to study the possibilities for proving the other inequality $\alpha_{X}(\tau, \omega) \leq \bar{s}$, which is more involved since lower estimates for local oscillations of erratic paths are usually more difficult to obtain than upper estimates. Roughly speaking, the classical strategy (see e.g. $[9,10,22,27,26])$ for dealing with such kind of issues relies on a fundamental general intuitive idea called the Berman's principle: "the more regular (smooth) is a path of a local time, the more irregular (erratic) is the corresponding path of the stochastic field associated with this local time". Thus, in our setting the classical strategy consists in showing that the local time of the field $X$ is jointly continuous and satisfies some Hölder regularity conditions. The proofs of these regularity results for the local time of $X$ require the latter field to have a local nondeterminism property, which imposes severe restrictions on the choice of the function $g$ in (1.1), for instance it has to be such that

$$
\liminf _{|\xi| \rightarrow+\infty}\left\{|\xi|^{s+N / 2}|g(\xi)|\right\}>0, \quad \text { for all } s \in(\bar{s}, 1) .
$$

Therefore, in our present article we have preferred to avoid making use of the classical strategy. This has led us to introduce in it a rather new strategy which makes an extensive use of Littlelwood-Paley methods in order to fully exploit the information on the Besov regularity of $\mathcal{F}^{-1}(g)$ provided by (1.10). Thanks to the latter strategy, we have obtained two theorems which show that (1.16) is valid under rather weak conditions on $g$. It is worth noting that these two theorems can easily be extended to non-stationary Gaussian fields having stationary increments. The main goal of the present section is to give their precise statements and explain them. Let us now state the first one of these two theorems.

Theorem 2.1 Assume that the condition $\left(\mathcal{H}_{0}\right)$ holds and that the function $g$ in (1.1) satisfies the following condition:

$\left(\mathcal{H}_{1}\right)$ There are a constant $a_{0} \in(0,1]$ and a positive integer $j_{0}$ such that, for every integer $j \geq j_{0}$, one has

$$
a_{0} g\left(2^{j+1} \eta\right) \leq g\left(2^{j} \eta\right), \quad \text { for almost all } \eta \in \mathcal{R}_{0}:=\left\{\eta \in \mathbb{R}^{N}: 2^{-1} \leq|\eta| \leq 2\right\} \text {. }
$$


Then, there exists $\Omega^{*}$ an event of probability 1 not depending $\tau$ such that (1.16) holds.

Actually the condition $\left(\mathcal{H}_{1}\right)$ is closely connected to the very useful characterization of Besov spaces through the Littlewood-Paley decomposition for tempered distributions (see Proposition 2.2 and Theorem 2.3 below). We mention in passing that any function in $L_{\text {loc }}^{1}\left(\mathbb{R}^{N}\right)$ which is bounded in vicinity of infinity by some polynomial function can be viewed as a tempered distribution (see e.g. [25] Chapter 1).

Proposition 2.2 Let $\varphi$ be a real-valued even function belonging to the Schwartz class $S\left(\mathbb{R}^{N}\right)$ (see e.g. [25] Chapter 1) whose Fourier transform $\widehat{\varphi}$ is an even compactly supported function with values in the interval $[0,1] \subset \mathbb{R}$, which further satisfies:

$$
\operatorname{Supp} \widehat{\varphi} \subseteq \mathcal{B}(0,1):=\left\{\xi \in \mathbb{R}^{N}:|\xi| \leq 1\right\}
$$

and

$$
\widehat{\varphi}(\xi)=1, \quad \text { for every } \xi \in \mathcal{B}\left(0,2^{-1}\right):=\left\{\xi \in \mathbb{R}^{N}:|\xi| \leq 2^{-1}\right\} .
$$

One denotes by $\left(\psi_{j}\right)_{j \in \mathbb{Z}_{+}}$the sequence of the real-valued even functions belonging to the Schwartz class $S\left(\mathbb{R}^{N}\right)$ defined through their Fourier transforms in the following way:

$$
\widehat{\psi}_{j}(\xi):=\widehat{\psi}_{0}\left(2^{-j} \xi\right):=\widehat{\varphi}\left(2^{-j-1} \xi\right)-\widehat{\varphi}\left(2^{-j} \xi\right), \quad \text { for all }(j, \xi) \in \mathbb{Z}_{+} \times \mathbb{R}^{N} \text {. }
$$

Then, each tempered distribution $f$ can be expressed in the space of the tempered distributions $S^{\prime}\left(\mathbb{R}^{N}\right)$ as:

$$
f=f * \varphi+\sum_{j=0}^{+\infty} f * \psi_{j},
$$

where "*" denotes the convolution product and the convergence of the series holds in the sense of the topology of $S^{\prime}\left(\mathbb{R}^{N}\right)$. Notice that the Littlewood-Paley components $f * \varphi$ and $f * \psi_{j}, j \in \mathbb{Z}_{+}$, are ${ }^{4}$ infinitely differentiable functions on $\mathbb{R}^{N}$ bounded by some polynomial functions.

The following theorem provides a very classical characterization of Besov spaces through Littlewood-Paley components; its proof can for instance be found in [8] Chapter 6 .

\footnotetext{
${ }^{4}$ More precisely, they can be identified with such functions.
} 
Theorem 2.3 Let $p \in[1,+\infty]$ and $s \in(0,+\infty)$ be arbitrary and fixed. A necessary and sufficient condition for a tempered distribution $f$ to be a function belonging to the Besov space $B_{p, \infty}^{s}\left(\mathbb{R}^{N}\right)$ is that its Littlewood-Paley components $f * \varphi$ and $f * \psi_{j}, j \in \mathbb{Z}_{+}$, belong to $L^{p}\left(\mathbb{R}^{N}\right)$ and satisfy

$$
\limsup _{j \rightarrow+\infty}\left\{2^{j s}\left\|f * \psi_{j}\right\|_{L^{p}\left(\mathbb{R}^{N}\right)}\right\}<+\infty
$$

\section{Remarks 2.4}

(i) The equality (2.5) is mainly a consequence of the equality

$$
\widehat{\varphi}(\xi)+\sum_{j=0}^{+\infty} \widehat{\psi}_{j}(\xi)=1, \quad \text { for all } \xi \in \mathbb{R}^{N},
$$

which easily results from (2.4) and (2.3).

(ii) It easily follows from (2.2), (2.3) and (2.4) that

$$
\operatorname{Supp} \widehat{\psi}_{j} \subseteq \mathcal{R}_{j}:=\left\{\xi \in \mathbb{R}^{N}: 2^{j-1} \leq|\xi| \leq 2^{j+1}\right\}, \quad \text { for all } j \in \mathbb{Z}_{+} .
$$

(iii) Combining Theorem 2.3 and Remark 1.1 one gets that

$$
\limsup _{j \rightarrow+\infty}\left\{2^{j s}\left\|\mathcal{F}^{-1}(g) * \psi_{j}\right\|_{L^{2}\left(\mathbb{R}^{N}\right)}\right\}<+\infty, \quad \text { for all } s \in(0, \bar{s}),
$$

and

$$
\limsup _{j \rightarrow+\infty}\left\{2^{j s}\left\|\mathcal{F}^{-1}(g) * \psi_{j}\right\|_{L^{2}\left(\mathbb{R}^{N}\right)}\right\}=+\infty, \quad \text { for all } s \in(\bar{s}, 1) .
$$

(iv) An important ingredient of the proof of Theorem 2.1 is the inequality

$$
2^{-N / 2}\left\|\mathcal{F}^{-1}(g) * \psi_{j+1}\right\|_{L^{2}\left(\mathbb{R}^{N}\right)} \leq\left\|\mathcal{F}^{-1}(g) * \psi_{j}\right\|_{L^{2}\left(\mathbb{R}^{N}\right)}, \quad \text { for all } j \geq j_{0}
$$

which results from the Plancherel formula, (2.4), the change of variable $\eta=2^{-j} \xi$, (2.7), (2.1), and the change of variable $\xi^{\prime}=2^{j+1} \eta$ :

$$
\begin{aligned}
& \left\|\mathcal{F}^{-1}(g) * \psi_{j}\right\|_{L^{2}\left(\mathbb{R}^{N}\right)}^{2}=(2 \pi)^{-N}\left\|\mathcal{F}\left(\mathcal{F}^{-1}(g) * \psi_{j}\right)\right\|_{L^{2}\left(\mathbb{R}^{N}\right)}^{2} \\
& =(2 \pi)^{-N} \int_{\mathbb{R}^{N}}\left|g(\xi) \widehat{\psi}_{0}\left(2^{-j} \xi\right)\right|^{2} d \xi=(2 \pi)^{-N} 2^{j N} \int_{\mathcal{R}_{0}}\left|g\left(2^{j} \eta\right) \widehat{\psi}_{0}(\eta)\right|^{2} d \eta \\
& \geq(2 \pi)^{-N} 2^{j N} \int_{\mathcal{R}_{0}}\left|g\left(2^{j+1} \eta\right) \widehat{\psi}_{0}(\eta)\right|^{2} d \eta \\
& =(2 \pi)^{-N} 2^{-N} \int_{\mathbb{R}^{N}}\left|g\left(\xi^{\prime}\right) \widehat{\psi}_{0}\left(2^{-j-1} \xi^{\prime}\right)\right|^{2} d \xi^{\prime}=2^{-N}\left\|\mathcal{F}^{-1}(g) * \psi_{j+1}\right\|_{L^{2}\left(\mathbb{R}^{N}\right)}^{2} .
\end{aligned}
$$


In order to state our second main result one needs to introduce, for every $n \in \mathbb{N}$, the function $g_{\nabla, n}$ defined as follows:

Definition 2.5 For every $n \in \mathbb{N}$, the real-valued even function $g_{\nabla, n} \in L^{2}\left(\mathbb{R}^{N}\right)$ is defined, for all $\xi \in \mathbb{R}^{N}$, as:

$$
g_{\nabla, n}(\xi):=\left\{\begin{array}{l}
g(\xi), \quad \text { if }|\xi| \geq 2^{n-1} \\
0, \quad \text { else }
\end{array}\right.
$$

where $g$ denotes the same function as in (1.1).

Remark 2.6 Observe that it follows from (2.11) and (2.7) that

$$
g \cdot \widehat{\psi}_{j}=g_{\nabla, n} \cdot \widehat{\psi}_{j}, \quad \text { for all } n \in \mathbb{N} \text { and } j \geq n
$$

and consequently that

$$
\mathcal{F}^{-1}(g) * \psi_{j}=\mathcal{F}^{-1}\left(g_{\nabla, n}\right) * \psi_{j}, \quad \text { for all } n \in \mathbb{N} \text { and } j \geq n \text {. }
$$

Therefore, Theorem 2.3 and (1.10) imply that

$$
\bar{s}=\sup \left\{s \in(0,1): \mathcal{F}^{-1}\left(g_{\nabla, n}\right) \in B_{2, \infty}^{s}\left(\mathbb{R}^{N}\right)\right\}, \quad \text { for every } n \in \mathbb{N} .
$$

We are now in a position to state our second main result.

Theorem 2.7 Assume that the condition $\left(\mathcal{H}_{0}\right)$ holds and that for some $n_{0} \in \mathbb{N}$ the function $\mathcal{F}^{-1}\left(g_{\nabla, n_{0}}\right)$ satisfies the following condition:

$\left(\mathcal{H}_{2}\right) \mathcal{F}^{-1}\left(g_{\nabla, n_{0}}\right) \in \bigcup_{p \in[4 / 3,2)} \bigcap_{s \in(0, \bar{s})} B_{p, \infty}^{s+\nu_{N, p}}\left(\mathbb{R}^{N}\right)$, where $\nu_{N, p}:=\frac{N(2-p)}{2 p}$.

Then, there exists $\Omega^{*}$ an event of probability 1 not depending $\tau$ such that (1.16) holds. 
Remark 2.8 Observe that (2.14) with $n=n_{0}$ is equivalent to the following two conditions on $\mathcal{F}^{-1}\left(g_{\nabla, n_{0}}\right)$ :

$$
\mathcal{F}^{-1}\left(g_{\nabla, n_{0}}\right) \in \bigcap_{s \in(0, \bar{s})} B_{2, \infty}^{s}\left(\mathbb{R}^{N}\right)
$$

and

$$
\mathcal{F}^{-1}\left(g_{\nabla, n_{0}}\right) \notin \bigcup_{s \in(\bar{s}, 1)} B_{2, \infty}^{s}\left(\mathbb{R}^{N}\right) .
$$

In fact the condition $\left(\mathcal{H}_{2}\right)$ in Theorem 2.7 is a bit stronger than (2.15). Indeed, one knows from e.g. Theorem 6.5.1 on page 153 in [8] that

$$
B_{p_{1}, \infty}^{s+\nu_{N, p_{1}}}\left(\mathbb{R}^{N}\right) \subseteq B_{p_{2}, \infty}^{s+\nu_{N, p_{2}}}\left(\mathbb{R}^{N}\right) \subset B_{2, \infty}^{s}\left(\mathbb{R}^{N}\right), \quad \text { for all } s>0 \text { and } 3 / 4 \leq p_{1} \leq p_{2}<2,
$$

and consequently that

$$
\bigcup_{p \in[4 / 3,2)} \bigcap_{s \in(0, \bar{s})} B_{p, \infty}^{s+\nu_{N, p}}\left(\mathbb{R}^{N}\right) \subset \bigcap_{s \in(0, \bar{s})} B_{2, \infty}^{s}\left(\mathbb{R}^{N}\right) .
$$

Before ending this section let us give an important corollary of Theorems 2.1 and 2.7 which concerns non-stationary Gaussian fields having stationary increments.

Corollary 2.9 Let $\{Y(t)\}_{t \in \mathbb{R}^{N}}$ be a centred real-valued Gaussian field with stationary increments of the general form:

$$
Y(t):=\int_{\mathbb{R}^{N}}\left(e^{-i t \cdot \xi}-1\right) \widetilde{g}(\xi) d \widehat{W}(\xi), \quad \text { for each } t \in \mathbb{R}^{N},
$$

where $\widetilde{g}$ is an arbitrary deterministic, even, real-valued, non-negative and Lebesgue measurable function such that

$$
\int_{\mathbb{R}^{N}} \min \left\{1,|\xi|^{2}\right\} \cdot|\widetilde{g}(\xi)|^{2} d \xi<+\infty .
$$

For all $n \in \mathbb{N}$, the function $\widetilde{g}_{\nabla, n}$ is defined by replacing in (2.11) $g$ by $\widetilde{g}$. In the same vein, the variogram $V_{Y}$ is defined by replacing in (1.6) $X$ by $Y$. One imposes to it the following condition:

$\left(\mathcal{H}_{0}^{\prime}\right)$ For some $\bar{s} \in(0,1)$, the inequality (1.8) and the equality (1.9) remain valid when $V_{X}(h)$ is replaced by $V_{Y}(h)$; 
which, among other things, implies that $Y$ has a modification with Hölder continuous paths to which it is identified. Also, one assumes that one of the following two conditions $\left(\mathcal{H}_{1}^{\prime}\right)$ and $\left(\mathcal{H}_{2}^{\prime}\right)$ is satisfied.

$\left(\mathcal{H}_{1}^{\prime}\right)$ The condition $\left(\mathcal{H}_{1}\right)$ (see Theorem 2.1) holds for the function $\widetilde{g}$ in place of the function $g$.

$\left(\mathcal{H}_{2}^{\prime}\right)$ For some $n_{0} \in \mathbb{N}$, the condition $\left(\mathcal{H}_{2}\right)$ (see Theorem 2.7) holds for the function $\widetilde{g}_{\nabla, n_{0}}$ in place of the function $g_{\nabla, n_{0}}$.

Then there exists $\Omega^{*}$ an event of probability 1 (not depending $\tau$ ) such that one has

$$
\alpha_{Y}(\tau, \omega)=\bar{s}, \quad \text { for all }(\tau, \omega) \in \mathbb{R}^{N} \times \Omega^{*},
$$

where $\alpha_{Y}(\cdot, \omega)$ denotes the pointwise Hölder exponent function of an arbitrary continuous path $Y(\cdot, \omega)$ of the field $Y$.

Proof of Corollary 2.9 It follows from (2.18) that, for all $t \in \mathbb{R}^{N}$, one has

$$
Y(t)=Z(t)+\tilde{X}(t)-\tilde{X}(0),
$$

where

$$
Z(t):=\int_{\{|\xi|<1\}}\left(e^{-i t \cdot \xi}-1\right) \widetilde{g}(\xi) d \widehat{W}(\xi)
$$

and

$$
\widetilde{X}(t):=\int_{\mathbb{R}^{N}} e^{-i t \cdot \xi} \widetilde{g}_{\nabla, 1}(\xi) d \widehat{W}(\xi) .
$$

Observe that one knows from (2.19) and the definition of the function $\widetilde{g}_{\nabla, 1}$ that this function belongs to $L^{2}\left(\mathbb{R}^{N}\right)$, which guarantees the existence of the stochastic integral in $(2.23)$.

Let us now show that the real-valued centred Gaussian field with stationary increments $\{Z(t)\}_{t \in \mathbb{R}^{N}}$ has a smooth modification. Using (2.22), (1.4), the inequality

$$
\left|e^{-i h \cdot \xi}-1\right| \leq|h| \cdot|\xi|, \quad \text { for all }(h, \xi) \in \mathbb{R}^{N} \times \mathbb{R}^{N}
$$

and (2.19), it follows that $V_{Z}(h):=\mathbb{E}\left(|Z(h)-Z(0)|^{2}\right)=\mathbb{E}\left(|Z(h)|^{2}\right)$, the variogram of $\{Z(t)\}_{t \in \mathbb{R}^{N}}$, satisfies

$$
V_{Z}(h) \leq c_{1}|h|^{2}, \quad \text { for all } h \in \mathbb{R}^{N},
$$


where the finite constant $c_{1}:=\int_{\{|\xi|<1\}}|\xi|^{2}|\widetilde{g}(\xi)|^{2} d \xi$. Thus, one can derive from the same arguments as in Remark 1.3 that the field $\{Z(t)\}_{t \in \mathbb{R}^{N}}$ has a modification, also denoted by $\{Z(t)\}_{t \in \mathbb{R}^{N}}$, whose paths are with probability 1 smooth functions: they are continuous on $\mathbb{R}^{N}$ and satisfy on each compact subset of it a Hölder condition of any arbitrary order $\gamma \in(0,1)$.

Next, notice that (2.21), the triangle inequality, (2.24), and $\left(\mathcal{H}_{0}^{\prime}\right)$ imply that the variogram $V_{\widetilde{X}}$ of the real-valued centred stationary Gaussian field $\{\tilde{X}(t)\}_{t \in \mathbb{R}^{N}}$ satisfies (1.8) and (1.9). Thus, in view of $\left(\mathcal{H}_{1}^{\prime}\right),\left(\mathcal{H}_{2}^{\prime}\right)$ and the definition of $\widetilde{g}_{\nabla, 1}$, one of the two Theorems 2.1 and 2.7 can be applied to the field $\{\widetilde{X}(t)\}_{t \in \mathbb{R}^{N}}$. Therefore, it turns out that (1.16) holds with $\alpha_{X}(\tau, \omega)$ replaced by $\alpha_{\tilde{X}}(\tau, \omega)$, where $\alpha_{\widetilde{X}}(\cdot, \omega)$ denotes the pointwise Hölder exponent function of any arbitrary continuous path $\widetilde{X}(\cdot, \omega)$ of the field $\{\tilde{X}(t)\}_{t \in \mathbb{R}^{N}}$. Then using (2.21) and the smoothness of paths of $\{Z(t)\}_{t \in \mathbb{R}^{N}}$, one obtains (2.20).

\section{Some auxiliary results and their proofs}

Lemma 3.1 Under the sole condition ${ }^{5}$ that the paths of the stationary Gaussian field $\{X(t)\}_{t \in \mathbb{R}^{N}}$ are almost surely continuous functions, there exists $\Omega_{1}^{*}$ an event of probability 1 such that one has on $\Omega_{1}^{*}$

$$
C_{1}^{*}:=\sup _{t \in \mathbb{R}^{N}}\left\{\frac{|X(t)|}{\sqrt{\log (3+|t|)}}\right\}<+\infty .
$$

Proof One denotes by $I_{0,0}$ the compact unit cube of $\mathbb{R}^{N}$, that is

$$
I_{0,0}:=[0,1]^{N}
$$

For every $k \in \mathbb{Z}^{N}$, let $\mu_{k}$ be the non-negative and finite random variable defined as:

$$
\mu_{k}:=\sup _{s \in I_{0,0}}|X(k+s)|
$$

Notice that the finiteness of $\mu_{k}$ follows from the continuity of paths of the field $X$, and that in turn it implies (see e.g. [21]) that the expectation $\mathbb{E}\left(\mu_{k}\right)$ is finite since $X$ is

\footnotetext{
${ }^{5}$ Recall that one knows from Remark 1.3 that it is enough that (1.8) be satisfied for the paths of $\{X(t)\}_{t \in \mathbb{R}^{N}}$ be almost surely continuous functions.
} 
Gaussian. Also, notice that the stationarity of the latter field entails that the random variables $\mu_{k}$ and $\mu_{0}$ have the same distribution. Thus, for every $k \in \mathbb{Z}^{N}$ and positive real-number $z$, one has

$$
\mathbb{P}\left(\left|\mu_{k}-\mathbb{E}\left(\mu_{0}\right)\right|>z\right)=\mathbb{P}\left(\left|\mu_{0}-\mathbb{E}\left(\mu_{0}\right)\right|>z\right) .
$$

Next, one sets $\sigma_{X}^{2}:=\mathbb{E}\left(|X(0)|^{2}\right)$. Observe that it follows from the stationarity of the field $X$ that, for all $t \in \mathbb{R}^{N}$, one has $\sigma_{X}^{2}=\mathbb{E}\left(|X(t)|^{2}\right)$, which clearly implies that $\sigma_{X}^{2}=\sup _{t \in I_{0,0}} \mathbb{E}\left(|X(t)|^{2}\right)$. Also, observe that obviously there is no restriction to assume that $\sigma_{X}>0$, since the field $X$ vanishes when $\sigma_{X}=0$ and then (3.1) is trivially satisfied. Next, using a Borell type inequality (see e.g. the inequality (3.2) on page 54 in [21] or [1]) one gets that

$$
\mathbb{P}\left(\left|\mu_{0}-\mathbb{E}\left(\mu_{0}\right)\right|>z\right) \leq 2 \exp \left(-2 z^{2} / \pi^{2} \sigma_{X}^{2}\right) .
$$

Next, combining (3.4) and (3.5), one obtains, for all $k \in \mathbb{Z}^{N}$, that

$$
\mathbb{P}\left(\left|\mu_{k}-\mathbb{E}\left(\mu_{0}\right)\right|>\pi \sigma_{X} \sqrt{N \log (3+|k|)}\right) \leq 2(3+|k|)^{-2 N}
$$

and consequently that

$$
\mathbb{E}\left(\sum_{k \in \mathbb{Z}^{N}} \mathbb{1}_{A_{k}}\right)=\sum_{k \in \mathbb{Z}^{N}} \mathbb{P}\left(A_{k}\right)<+\infty
$$

where $\mathbb{1}_{A_{k}}$ is the indicator function of the event

$$
A_{k}:=\left\{\left|\mu_{k}-\mathbb{E}\left(\mu_{0}\right)\right|>\pi \sigma_{X} \sqrt{N \log (3+|k|)}\right\}, \quad \text { for each } k \in \mathbb{Z}^{N} .
$$

Next, observe that (3.6) implies that the non-negative random variable $\sum_{k \in \mathbb{Z}^{N}} \mathbb{1}_{A_{k}}$ is almost surely finite. Therefore, there exists $\Omega_{1}^{*}$ an event of probability 1 such that, for each $\omega \in \Omega_{1}^{*}$, the inequality

$$
\left|\mu_{k}(\omega)-\mathbb{E}\left(\mu_{0}\right)\right|>\pi \sigma_{X} \sqrt{N \log (3+|k|)}
$$

may hold only for a finite number of indices $k \in \mathbb{Z}^{N}$. Thus, one has that

$$
\sup _{k \in \mathbb{Z}^{N}}\left\{\frac{\mu_{k}(\omega)}{\sqrt{\log (3+|k|)}}\right\}<+\infty \text {. }
$$

Finally, combining (3.7) with (3.3), (3.2) and the inequality $|s| \leq \sqrt{N}$, for all $s \in I_{0,0}$, one gets (3.1). 
Definition 3.2 Let $\psi_{0} \in S\left(\mathbb{R}^{N}\right)$ be the same function as in Proposition 2.2. For all $j \in \mathbb{Z}_{+}$and $k \in \mathbb{Z}^{N}$, one denotes by $\chi_{j, k}$ the real-valued random variable defined almost surely as the pathwise Lebesgue integral:

$$
\chi_{j, k}:=\int_{\mathbb{R}^{N}} 2^{j N} \psi_{0}\left(2^{j} t-k\right) X(t) d t .
$$

Notice that, in view of Lemma 3.1, the integral in (3.8) exists almost surely and is finite under the sole condition that the paths of the stationary Gaussian field $\{X(t)\}_{t \in \mathbb{R}^{N}}$ are almost surely continuous functions.

Lemma 3.3 Under the sole condition that the paths of the stationary Gaussian field $\{X(t)\}_{t \in \mathbb{R}^{N}}$ are almost surely continuous functions, for all $j \in \mathbb{Z}_{+}$and $k \in \mathbb{Z}^{N}$, the random variable $\chi_{j, k}$ can be almost surely expressed as:

$$
\chi_{j, k}=\int_{\mathbb{R}^{N}} \mathcal{F}\left(2^{j N} \psi_{0}\left(2^{j} \cdot-k\right)\right)(\xi) g(\xi) d \widehat{W}(\xi)=\int_{\mathbb{R}^{N}} e^{-i 2^{-j} k \cdot \xi} \widehat{\psi}_{j}(\xi) g(\xi) d \widehat{W}(\xi),
$$

where $g$ is the same function as in (1.1). Thus, $\left\{\chi_{j, k}\right\}_{(j, k) \in \mathbb{Z}_{+} \times \mathbb{Z}^{N}}$ is a centred real-valued Gaussian process. Moreover, it follows from (3.9) and the isometry property (1.4) that, for all $(j, k) \in \mathbb{Z}_{+} \times \mathbb{Z}^{N}$, the standard deviation $\sigma\left(\chi_{j, k}\right):=\sqrt{\mathbb{E}\left(\left|\chi_{j, k}\right|^{2}\right)}$ of $\chi_{j, k}$, satisfies

$$
\sigma\left(\chi_{j, k}\right)=\left\|g \cdot \widehat{\psi}_{j}\right\|_{L^{2}\left(\mathbb{R}^{N}\right)}=(2 \pi)^{N / 2}\left\|\mathcal{F}^{-1}(g) * \psi_{j}\right\|_{L^{2}\left(\mathbb{R}^{N}\right)} .
$$

Proof Throughout the proof $j \in \mathbb{Z}_{+}$and $k \in \mathbb{Z}^{N}$ are arbitrary and fixed. For each $n \in \mathbb{Z}_{+}$, one denotes by $\left\{F_{n}(t)\right\}_{t \in \mathbb{R}^{N}}$ the centred real-valued Gaussian field with piecewise constant paths defined, for every $t \in \mathbb{R}^{N}$, as:

$$
F_{n}(t):=2^{j N} \sum_{|p| \leq 4^{n}} \psi_{0}\left(2^{j} d_{n, p}-k\right) X\left(d_{n, p}\right) \mathbb{1}_{I_{n, p}}(t)
$$

where, for all $p=\left(p_{1}, \ldots, p_{N}\right) \in \mathbb{Z}^{N}$, the dyadic vector $d_{n, p}$ and the dyadic cube $I_{n, p}$ are such that

$$
d_{n, p}:=2^{-n} p=\left(2^{-n} p_{1}, \ldots, 2^{-n} p_{N}\right)
$$

and

$$
I_{n, p}:=\prod_{l=1}^{N}\left[2^{-n} p_{l}, 2^{-n}\left(p_{l}+1\right)\right]
$$


Let us first show that one has almost surely

$$
\chi_{j, k}=\lim _{n \rightarrow+\infty} \int_{\mathbb{R}^{N}} F_{n}(t) d t=\lim _{n \rightarrow+\infty} 2^{(j-n) N} \sum_{|p| \leq 4^{n}} \psi_{0}\left(2^{j} d_{n, p}-k\right) X\left(d_{n, p}\right) .
$$

Using (3.11), the continuity of the function $\psi_{0}$ and that of the paths of the field $X$, one has

$$
2^{j N} \psi_{0}\left(2^{j} t-k\right) X(t)=\lim _{n \rightarrow+\infty} F_{n}(t), \quad \text { for almost all } t \in \mathbb{R}^{N} .
$$

Moreover, $\left|F_{n}(t)\right|$ can be bounded independently on $n$ as follows:

$$
\left|F_{n}(t)\right| \leq G(t), \quad \text { for all } n \in \mathbb{Z}_{+} \text {and almost all } t \in \mathbb{R}^{N},
$$

where the $\mathbb{R}_{+}$-valued stochastic process $\{G(t)\}_{t \in \mathbb{R}^{N}}$ is given, for any $t \in \mathbb{R}^{N}$, by

$$
G(t):=2^{j N} \sum_{q \in \mathbb{Z}^{N}} \sup _{s \in I_{0, q}}\left|\psi_{0}\left(2^{j} s-k\right) X(s)\right| \mathbb{1}_{I_{0, q}}(t) .
$$

Next, notice that using Lemma 3.1 and the fact that the function $\psi_{0}\left(2^{j} \cdot-k\right)$ belongs to $S\left(\mathbb{R}^{N}\right)$, one gets that

$$
\int_{\mathbb{R}^{N}} G(t) d t=2^{j N} \sum_{q \in \mathbb{Z}^{N}} \sup _{s \in I_{0, q}}\left|\psi_{0}\left(2^{j} s-k\right) X(s)\right|<+\infty,
$$

which means that the paths of the field $\{G(t)\}_{t \in \mathbb{R}^{N}}$ belong to $L^{1}\left(\mathbb{R}^{N}\right)$. Thus, in view of (3.15) and (3.16) the dominated convergence Theorem entails that (3.14) is satisfied.

On the other hand, (3.11) and (1.1) imply that

$$
\begin{aligned}
& \int_{\mathbb{R}^{N}} F_{n}(t) d t \\
& =2^{j N} \int_{\mathbb{R}^{N}}\left(2^{-n N} \sum_{|p| \leq 4^{n}} e^{-i d_{n, p} \cdot \xi} \psi_{0}\left(2^{j} d_{n, p}-k\right)\right) g(\xi) d \widehat{W}(\xi) \\
& =2^{j N} \int_{\mathbb{R}^{N}}\left(\int_{\mathbb{R}^{N}} \Phi_{n}(t, \xi) d t\right) g(\xi) d \widehat{W}(\xi),
\end{aligned}
$$

where, for all $(t, \xi) \in \mathbb{R}^{N} \times \mathbb{R}^{N}$,

$$
\Phi_{n}(t, \xi):=\sum_{|p| \leq 4^{n}} e^{-i d_{n, p} \cdot \xi} \psi_{0}\left(2^{j} d_{n, p}-k\right) \mathbb{1}_{I_{n, p}}(t) .
$$


Thus, in view of (3.14), (3.18) and the isometry property (1.4), in order to derive (3.9) it is enough to show that

$$
\lim _{n \rightarrow+\infty} \int_{\mathbb{R}^{N}}\left|\int_{\mathbb{R}^{N}}\left(e^{-i t \cdot \xi} \psi_{0}\left(2^{j} t-k\right)-\Phi_{n}(t, \xi)\right) d t\right|^{2} \times|g(\xi)|^{2} d \xi=0 .
$$

The first step for proving (3.20) consists in showing that

$$
\lim _{n \rightarrow+\infty} \int_{\mathbb{R}^{N}}\left|e^{-i t \cdot \xi} \psi_{0}\left(2^{j} t-k\right)-\Phi_{n}(t, \xi)\right| d t=0, \quad \text { for all } \xi \in \mathbb{R}^{N} .
$$

Using (3.19), the continuity of the function $\psi_{0}$ and that of the exponential function, it can easily be seen that, for all $\xi \in \mathbb{R}^{N}$ and for almost all $t \in \mathbb{R}^{N}$, the integrand in (3.21), denoted by $R_{n}(t, \xi)$, converges to 0 when $n \rightarrow+\infty$, and satisfies, for every $n \in \mathbb{Z}_{+}$,

$$
R_{n}(t, \xi):=\left|e^{-i t \cdot \xi} \psi_{0}\left(2^{j} t-k\right)-\Phi_{n}(t, \xi)\right| \leq H(t),
$$

where $H$ denotes the function in $L^{1}\left(\mathbb{R}^{N}\right)$ defined, for each $t \in \mathbb{R}^{N}$, as:

$$
H(t):=\left|\psi_{0}\left(2^{j} t-k\right)\right|+\sum_{q \in \mathbb{Z}^{N}} \sup _{s \in I_{0, q}}\left|\psi_{0}\left(2^{j} s-k\right)\right| \mathbb{1}_{I_{0, q}}(t) .
$$

Thus, applying the dominated convergence Theorem, one gets (3.21). It clearly implies that, for all $\xi \in \mathbb{R}^{N}$, the integrand in (3.20), denoted by $A_{n}(\xi)$, converges to 0 when $n \rightarrow+\infty$. Thus, (3.20) results from the dominated convergence Theorem, since one has, for every $(n, \xi) \in \mathbb{Z}_{+} \times \mathbb{R}^{N}, A_{n}(\xi) \leq\left\|R_{n}(\cdot, \xi)\right\|_{L^{1}\left(\mathbb{R}^{N}\right)}^{2}|g(\xi)|^{2} \leq\|H\|_{L^{1}\left(\mathbb{R}^{N}\right)}^{2}|g(\xi)|^{2}$ and the function $|g|^{2}$ belongs to $L^{1}\left(\mathbb{R}^{N}\right)$.

\section{Remarks 3.4}

(i) Let $I \subset \mathbb{R}^{N}$ be an arbitrary dyadic cube, that is there exists a unique couple $\left(j_{I}, k_{I}\right)=\left(j_{I}, k_{I, 1}, \ldots, k_{I, N}\right) \in \mathbb{Z}_{+} \times \mathbb{Z}^{N}$ such that

$$
I=I_{J_{I}, k_{I}}:=\prod_{l=1}^{N}\left[2^{-j_{I}} k_{I, l}, 2^{-j_{I}}\left(k_{I, l}+1\right)\right] ;
$$

for the sake of simplicity the random variable $\chi_{j_{I}, k_{I}}$ will often be denoted by $\chi_{I}$.

(ii) For each $j \in \mathbb{Z}_{+}$and $\tau=\left(\tau_{1}, \ldots, \tau_{N}\right) \in \mathbb{R}^{N}$, one sets

$$
k_{j}(\tau):=\left(\left[2^{j} \tau_{1}\right], \ldots,\left[2^{j} \tau_{N}\right]\right)
$$

where $[\cdot]$ is the integer part function. It is clear that $\tau$ belongs to the dyadic cube $I_{j, k_{j}(\tau)}$. 
Lemma 3.5 One assumes that the paths of the stationary Gaussian field $\{X(t)\}_{t \in \mathbb{R}^{N}}$ are almost surely continuous functions. For all $\tau \in \mathbb{R}^{N}$ and $\rho \in(0,1]$, the non-negative random variable $\mathcal{M}_{\chi}(\tau, \rho)$ is defined as:

$$
\mathcal{M}_{\chi}(\tau, \rho):=\sup \left\{\left|\chi_{I}\right|: I \text { dyadic cube s.t. } I \subseteq \mathcal{B}(\tau, \rho)\right\}
$$

where $\mathcal{B}(\tau, \rho):=\left\{t \in \mathbb{R}^{N}:|t-\tau| \leq \rho\right\}$. Let $\Omega_{1}^{*}$ be the same event of probability 1 (not depending on $\tau$ ) as in Lemma 3.1. Then the inequality

$$
\left\|\psi_{0}\right\|_{L^{1}\left(\mathbb{R}^{N}\right)} \limsup _{r>0, r \rightarrow 0}\left\{r^{-\alpha} \operatorname{Osc}_{X}(\tau, r, \omega)\right\} \geq \limsup _{r>0, r \rightarrow 0}\left\{r^{-\alpha} \mathcal{M}_{\chi}\left(\tau, r^{\gamma}, \omega\right)\right\}
$$

holds for all $\alpha \in[0,1], \gamma>1$ and $\omega \in \Omega_{1}^{*}$.

Proof Let $\tau \in \mathbb{R}^{N}, \gamma \in(1,+\infty)$ and $r \in(0,1]$ be arbitrary, and let $I_{j, k}, j \in \mathbb{Z}_{+}$and $k=\left(k_{1}, \ldots, k_{N}\right) \in \mathbb{Z}^{N}$, be any dyadic cube such that

$$
I_{j, k}:=\prod_{l=1}^{N}\left[2^{-j} k_{l}, 2^{-j}\left(k_{l}+1\right)\right] \subseteq \mathcal{B}\left(\tau, r^{\gamma}\right) .
$$

As usual, one sets $d_{j, k}:=2^{-j} k$. In view of (3.25), one necessarily has that

$$
2 r^{\gamma} \geq N^{1 / 2} 2^{-j}=\operatorname{Diam}\left(I_{j, k}\right):=\sup \left\{\left|x^{\prime}-x^{\prime \prime}\right|:\left(x^{\prime}, x^{\prime \prime}\right) \in I_{j, k}^{2}\right\}
$$

and

$$
\left|\tau-d_{j, k}\right| \leq r^{\gamma} \leq r .
$$

Using (3.8), the change of variable $s=2^{j} t-k$ and the equality $\int_{\mathbb{R}^{N}} \psi_{0}(s) d s=0$ (which results from $(2.7))$, one gets that

$$
\left|\chi_{j, k}\right| \leq \int_{\mathbb{R}^{N}}\left|\psi_{0}(s)\right|\left|X\left(d_{j, k}+2^{-j} s\right)-X\left(d_{j, k}\right)\right| d t
$$

Next, observe that if $s \in A(r):=\left\{s \in \mathbb{R}^{N}:|s| \leq r^{(1-\gamma) / 2}\right\}$ then (3.26), (3.27) and the triangle inequality imply that

$$
\left|d_{j, k}+2^{-j} s-\tau\right| \leq r^{\gamma}+2 r^{(1+\gamma) / 2} \leq r
$$


where the last inequality holds for each $r$ small enough since $\gamma>1$. Thus, when $r$ is small enough, it follows from (3.27), (3.29) and (1.14) that

$$
\int_{A(r)}\left|\psi_{0}(s)\right|\left|X\left(d_{j, k}+2^{-j} s\right)-X\left(d_{j, k}\right)\right| d t \leq\left\|\psi_{0}\right\|_{L^{1}\left(\mathbb{R}^{N}\right)} \operatorname{Osc}_{X}(\tau, r) .
$$

Next observe that using (3.27), the triangle inequality and (3.1), one has on the event of probability $1 \Omega_{1}^{*}$, for all $s \in \mathbb{R}^{N}$ with $|s| \geq 1$,

$$
\left|X\left(d_{j, k}+2^{-j} s\right)-X\left(d_{j, k}\right)\right| \leq 2 C_{1}^{*} \sqrt{\log (4+|\tau|+|s|)} \leq C_{1}^{\prime}|s|,
$$

where $C_{1}^{*}$ is the same finite random variable as in $(3.1)$, and $C_{1}^{\prime}$ is a finite random variable only depending on $C_{1}^{*}$ and $|\tau|$. On the other hand, the fact that $\psi_{0}$ belongs to $S\left(\mathbb{R}^{N}\right)$ implies that, for any fixed $L \in(N+1,+\infty)$, there exists a finite constant $c_{2}(L)$ such that, for all $s \in \mathbb{R}^{N}$ with $|s| \geq 1$, one has

$$
\left|\psi_{0}(s)\right| \leq c_{2}(L)|s|^{-L}
$$

Thus, setting $\bar{A}(r):=\mathbb{R}^{N} \backslash A(r)=\left\{s \in \mathbb{R}^{N}:|s|>r^{(1-\gamma) / 2}\right\}$ (notice that $r^{(1-\gamma) / 2} \geq 1$ ), and using (3.32), (3.31), and the change of variable $u=r^{(\gamma-1) / 2} s$, one obtains that

$$
\begin{aligned}
& \int_{\bar{A}(r)}\left|\psi_{0}(s)\right|\left|X\left(d_{j, k}+2^{-j} s\right)-X\left(d_{j, k}\right)\right| d t \\
& \leq C_{1}^{\prime} c_{2}(L) \int_{\bar{A}(r)}|s|^{1-L} d s=C_{1}^{\prime} c_{2}(L) r^{N(1-\gamma) / 2} \int_{\{|u|>1\}}\left|r^{(1-\gamma) / 2} u\right|^{1-L} d u \\
& =\left(C_{1}^{\prime} c_{2}(L) \int_{\{|u|>1\}}|u|^{1-L} d u\right) r^{(L-N-1)(\gamma-1) / 2}
\end{aligned}
$$

Finally, taking $L \in(N+1,+\infty)$ large enough so that $(L-N-1)(\gamma-1) / 2>1$, and putting together (3.23), (3.25), (3.28), (3.30) and (3.33), it follows that (3.24) holds.

\section{Proofs of the two main theorems}

Proof of Theorem 2.1 Let $\varepsilon>0$ be arbitrarily small and fixed, and let $n_{0}(\varepsilon) \in \mathbb{N}$ be such that

$$
n_{0}(\varepsilon) \varepsilon>N
$$


As usual, for all $j \in \mathbb{Z}_{+}$and $k=\left(k_{1}, \ldots, k_{N}\right) \in \mathbb{Z}^{N}$, the dyadic cube $I_{j, k}$ having a Lebesgue measure $\lambda\left(I_{j, k}\right)=2^{-j N}$ is defined as:

$$
I_{j, k}:=\prod_{l=1}^{N}\left[2^{-j} k_{l}, 2^{-j}\left(k_{l}+1\right)\right] .
$$

For each integer $j \geq 2 n_{0}(\varepsilon)$ and $k \in \mathbb{Z}^{N}$, one denotes by $\mathcal{A}_{j, k}^{\varepsilon}$ the finite set of "ancestors" of $I_{j, k}$ consisting in the $n_{0}(\varepsilon)$ dyadic cubes $\mathcal{J}_{0}, \mathcal{J}_{1}, \ldots, \mathcal{J}_{n_{0}(\varepsilon)-1}$ such that

$$
I_{j, k}=\mathcal{J}_{0} \subset \mathcal{J}_{1} \subset \ldots \subset \mathcal{J}_{n_{0}(\varepsilon)-1} \text { and } \lambda\left(\mathcal{J}_{n}\right)=2^{-(j-2 n) N} \text {, for all } n \in\left\{0,1, \ldots, n_{0}(\varepsilon)-1\right\} .
$$

We are now going to show that the real-valued centred Gaussian random variables $\chi_{\mathfrak{J}_{0}}, \chi_{\mathfrak{I}_{1}}, \ldots, \chi_{\mathfrak{J}_{n_{0}(\varepsilon)-1}}$ (see Definition 3.2, Lemma 3.3 and $(i)$ in Remarks 3.4) are independent. In view of the fact that they form a Gaussian vector, it is enough to prove that any two distinct random variables of them have a vanishing covariance. Let $n^{\prime}, n^{\prime \prime} \in\left\{0,1, \ldots, n_{0}(\varepsilon)-1\right\}$ be arbitrary and such that $n^{\prime} \neq n^{\prime \prime}$, and let $k^{\prime}, k^{\prime \prime} \in \mathbb{Z}^{N}$ be such that $\mathcal{J}_{n^{\prime}}=I_{j-2 n^{\prime}, k^{\prime}}$ and $\mathcal{J}_{n^{\prime \prime}}=I_{j-2 n^{\prime \prime}, k^{\prime \prime}}$. Therefore, one has that $\chi_{\mathfrak{J}_{n^{\prime}}}=\chi_{j-2 n^{\prime}, k^{\prime}}$ and $\chi_{\mathfrak{J}_{n^{\prime \prime}}}=\chi_{j-2 n^{\prime \prime}, k^{\prime \prime}}$. Then, one knows from (3.9) that

$$
\chi_{\mathfrak{J}_{n^{\prime}}}=\int_{\mathbb{R}^{N}} \exp \left(-i 2^{-\left(j-2 n^{\prime}\right)} k^{\prime} \cdot \xi\right) \widehat{\psi}_{j-2 n^{\prime}}(\xi) g(\xi) d \widehat{W}(\xi)
$$

and

$$
\chi_{\mathfrak{J}_{n^{\prime \prime}}}=\int_{\mathbb{R}^{N}} \exp \left(-i 2^{-\left(j-2 n^{\prime \prime}\right)} k^{\prime \prime} \cdot \xi\right) \widehat{\psi}_{j-2 n^{\prime \prime}}(\xi) g(\xi) d \widehat{W}(\xi) .
$$

Thus, using the isometry property (1.5) of the stochastic integral $\int_{\mathbb{R}^{N}}(\cdot) d \widehat{W}$ and $(2.7)$, one gets that

$$
\begin{aligned}
& \operatorname{Cov}\left(\chi_{\mathfrak{I}_{n^{\prime}}}, \chi_{\mathfrak{J}_{n^{\prime \prime}}}\right) \\
& =\int_{\mathbb{R}^{N}} \exp \left(i\left(2^{-\left(j-2 n^{\prime}\right)} k^{\prime}-2^{-\left(j-2 n^{\prime \prime}\right)} k^{\prime \prime}\right) \cdot \xi\right) \widehat{\psi}_{j-2 n^{\prime}}(\xi) \widehat{\psi}_{j-2 n^{\prime \prime}}(\xi)|g(\xi)|^{2} d \xi=0,
\end{aligned}
$$

which shows that $\chi_{\mathfrak{I}_{0}}, \chi_{\mathfrak{I}_{1}}, \ldots, \chi_{\mathfrak{J}_{n_{0}(\varepsilon)-1}}$ are independent.

Next, for every integer $j \geq 2 n_{0}(\varepsilon)$ and $k \in \mathbb{Z}^{N}$, one denotes by $A_{j, k}^{\varepsilon}$ the event defined as:

$$
A_{j, k}^{\varepsilon}:=\bigcap_{\mathcal{J} \in \mathcal{A}_{j, k}^{\varepsilon}}\left\{\left|\chi_{\mathcal{J}}\right|<2^{-j \varepsilon} \sigma\left(\chi_{\mathcal{J}}\right)\right\},
$$


where $\sigma\left(\chi_{\mathfrak{J}}\right)$ is the standard deviation of $\chi_{\mathfrak{J}}$. Using the independence and the Gaussianity of the $n_{0}(\varepsilon)$ random variables $\chi_{\mathcal{J}}$ in (4.4), one gets that

$$
\mathbb{P}\left(A_{j, k}^{\varepsilon}\right) \leq 2^{-j n_{0}(\varepsilon) \varepsilon} .
$$

Next, for each $p \in \mathbb{Z}^{N}$ and integer $j \geq 2 n_{0}(\varepsilon)$, let $\mathcal{K}_{p, j}$ be the finite subset of $\mathbb{Z}^{N}$ of cardinality $2^{j N}$ defined as

$$
\mathcal{K}_{p, j}:=2^{j} p+\mathcal{K}_{j}:=\left\{2^{j} p+l: l \in \mathcal{K}_{j}\right\}
$$

where $\mathcal{K}_{j}:=\left\{0, \ldots, 2^{j}-1\right\}^{N}$. Notice that it follows from (4.5), (4.1) and the equality $\operatorname{Card}\left(\mathcal{K}_{p, j}\right)=2^{N}$ that, for each fixed $p \in \mathbb{Z}^{N}$, one has

$$
\sum_{j=2 n_{0}(\varepsilon)}^{+\infty} \mathbb{P}\left(\bigcup_{k \in \mathcal{K}_{p, j}} A_{j, k}^{\varepsilon}\right) \leq \sum_{j=2 n_{0}(\varepsilon)}^{+\infty} \sum_{k \in \mathcal{K}_{p, j}} \mathbb{P}\left(A_{j, k}^{\varepsilon}\right) \leq \sum_{j=2 n_{0}(\varepsilon)}^{+\infty} 2^{-j\left(n_{0}(\varepsilon) \varepsilon-N\right)}<+\infty .
$$

Thus, denoting by $\bar{A}_{j, k}^{\varepsilon}$ the complement of the event $A_{j, k}^{\varepsilon}$ defined in (4.4), that is

$$
\bar{A}_{j, k}^{\varepsilon}:=\bigcup_{\mathcal{J} \in \mathcal{A}_{j, k}^{\varepsilon}}\left\{\left|\chi_{\mathcal{J}}\right| \geq 2^{-j \varepsilon} \sigma\left(\chi_{\mathfrak{J}}\right)\right\}
$$

it results from the Borel-Cantelli Lemma that

$$
\mathbb{P}\left(\bigcup_{J=2 n_{0}(\varepsilon)}^{+\infty} \bigcap_{j=J}^{+\infty} \bigcap_{k \in \mathcal{K}_{p, j}} \bar{A}_{j, k}^{\varepsilon}\right)=1
$$

Since $p \in \mathbb{Z}^{N}$ is arbitrary and $\mathbb{Z}^{N}$ is a countable set, (4.8) implies that the probability of the event

$$
\Omega_{2, \varepsilon}^{*}:=\bigcap_{p \in \mathbb{Z}^{N}} \bigcup_{J=2 n_{0}(\varepsilon)}^{+\infty} \bigcap_{j=J}^{+\infty} \bigcap_{k \in \mathcal{K}_{p, j}} \bar{A}_{j, k}^{\varepsilon}
$$

is equal to 1 . Observe that it results from (4.9), (4.7) and (3.22) that: for every $\tau \in \mathbb{R}^{N}$ and for each $\omega \in \Omega_{2, \varepsilon}^{*}$, there exists an integer $\bar{J}\left(k_{0}(\tau), \omega\right) \geq 2 n_{0}(\varepsilon)$ such that

$$
\max \left\{\left|\chi_{\mathcal{J}}(\omega)\right|: \mathcal{J} \in \mathcal{A}_{j, k_{j}(\tau)}^{\varepsilon}\right\} \geq 2^{-j \varepsilon} \min \left\{\sigma\left(\chi_{\mathcal{J}}\right): \mathcal{J} \in \mathcal{A}_{j, k_{j}(\tau)}^{\varepsilon}\right\}, \quad \text { for all } j \geq \bar{J}\left(k_{0}(\tau), \omega\right) \text {. }
$$

Next, let $c_{0}$ be the positive constant defined as $c_{0}:=2^{2 n_{0}(\varepsilon)-2} \sqrt{N}$. One knows from (4.3) and $(i i)$ in Remarks 3.4 that, for any integer $j \geq 2 n_{0}(\varepsilon)$ and for all dyadic cube $\mathcal{J} \in \mathcal{A}_{j, k_{j}(\tau)}^{\varepsilon}$, one has $\mathcal{J} \subset \mathcal{B}\left(\tau, c_{0} 2^{-j}\right)$. Thus, it follows from (4.10) and (3.23), that

$$
\mathcal{M}_{\chi}\left(\tau, c_{0} 2^{-j}, \omega\right) \geq 2^{-j \varepsilon} \min \left\{\sigma\left(\chi_{\mathcal{J}}\right): \mathcal{J} \in \mathcal{A}_{j, k_{j}(\tau)}^{\varepsilon}\right\}, \quad \text { for all } j \geq \bar{J}\left(k_{0}(\tau), \omega\right) \text {. }
$$


Next, setting $c_{1}:=\left(2^{3-2 n_{0}(\varepsilon)} \pi\right)^{N / 2} a_{0}^{2 n_{0}(\varepsilon)-2}$, one can derive from $(3.10),(2.10)$ and (4.11) that

$$
\mathcal{M}_{\chi}\left(\tau, c_{0} 2^{-j}, \omega\right) \geq c_{1} 2^{-j \varepsilon}\left\|\mathcal{F}^{-1}(g) * \psi_{j}\right\|_{L^{2}\left(\mathbb{R}^{N}\right)}, \quad \text { for all } j \geq \bar{J}\left(k_{0}(\tau), \omega\right)+j_{0} .
$$

Thus, it follows from (2.9) that

$$
\limsup _{j \rightarrow+\infty}\left\{2^{j(s+\varepsilon)} \mathcal{M}_{\chi}\left(\tau, c_{0} 2^{-j}, \omega\right)\right\}=+\infty, \quad \text { for all }(s, \tau, \omega) \in(\bar{s}, 1) \times \mathbb{R}^{N} \times \Omega_{2, \varepsilon}^{*} .
$$

Therefore, one has

$\limsup _{r>0, r \rightarrow 0}\left\{r^{-(s+\varepsilon) /(1-\varepsilon)} \mathcal{M}_{\chi}\left(\tau, r^{1 /(1-\varepsilon)}, \omega\right)\right\}=+\infty, \quad$ for all $(s, \tau, \omega) \in(\bar{s}, 1) \times \mathbb{R}^{N} \times \Omega_{2, \varepsilon}^{*}$.

Next, in view of the fact that $\varepsilon$ is arbitrarily small, one can assume without any restriction that it belongs to the interval $\left(0, \varepsilon_{1}\right)$, where $\varepsilon_{1}:=(1-\bar{s}) / 3$. Thus, one has that $(\bar{s}+2 \varepsilon) /(1-\varepsilon) \in(0,1)$. Then, (3.24) with $\alpha=(\bar{s}+2 \varepsilon) /(1-\varepsilon)$ and $\gamma=1 /(1-\varepsilon)$, and (4.12) with $s=\bar{s}+\varepsilon$, imply that

$$
\limsup _{r>0, r \rightarrow 0}\left\{r^{-(\bar{s}+2 \varepsilon) /(1-\varepsilon)} \operatorname{Osc}_{X}(\tau, r, \omega)\right\}=+\infty, \quad \text { for all }(\tau, \omega) \in \mathbb{R}^{N} \times \Omega_{3, \varepsilon}^{*},
$$

where $\Omega_{3, \varepsilon}^{*}$ is the event of probability 1 , not depending on $\tau$, defined as $\Omega_{3, \varepsilon}^{*}:=\Omega_{2, \varepsilon}^{*} \cap \Omega_{1}^{*}$. Next, combining (1.13) with (4.13), one obtains that

$$
\alpha_{X}(\tau, \omega) \leq \frac{\bar{s}+2 \varepsilon}{1-\varepsilon}, \quad \text { for all }(\tau, \omega) \in \mathbb{R}^{N} \times \Omega_{3, \varepsilon}^{*} .
$$

Next, one denotes by $\Omega_{3}^{*}$ the event of probability 1 , not depending on $\tau$, defined as

$$
\Omega_{3}^{*}:=\bigcap_{\varepsilon \in \mathbb{Q} \cap\left(0, \varepsilon_{1}\right)} \Omega_{3, \varepsilon}^{*},
$$

where $\mathbb{Q}$ is the countable set of the rational numbers. In view of (4.14), using the equality

$$
\lim _{\varepsilon>0, \varepsilon \rightarrow 0} \frac{\bar{s}+2 \varepsilon}{1-\varepsilon}=\bar{s}
$$

one gets that

$$
\alpha_{X}(\tau, \omega) \leq \bar{s} \quad \text { for all }(\tau, \omega) \in \mathbb{R}^{N} \times \Omega_{3}^{*} .
$$

Finally, combining (4.15) and Remark 1.3, it turns out that (1.16) holds when $\Omega^{*}:=\Omega_{3}^{*}$. 
From now on one focuses on Theorem 2.7. In order to derive it, first one needs to introduce some additional notations and to obtain some further preliminary results. Let $\varepsilon>0$ be arbitrarily small and fixed. For all $j \in \mathbb{N}$, the integer $\delta_{j}(\varepsilon) \geq 2$ is such that

$$
\delta_{j}(\varepsilon):=\left[2^{j \varepsilon}\right]+1
$$

where $[\cdot]$ is the integer part function. One denotes by $\mathcal{S}_{j}(\varepsilon)$ the finite subset of $\mathbb{Z}^{N}$ defined as:

$$
\mathcal{S}_{j}(\varepsilon)=\left\{0,1, \ldots,\left[2^{j(1-\varepsilon)}\right]+1\right\}^{N}
$$

One clearly has

$$
2^{j(1-\varepsilon) N} \leq \operatorname{Card}\left(\mathcal{S}_{j}(\varepsilon)\right) \leq 3^{N} 2^{j(1-\varepsilon) N} .
$$

For each $m \in \mathbb{Z}^{N}$ and $n \in \mathcal{S}_{j}$, the finite subset $\Lambda_{n, j}^{m}(\varepsilon)$ of $\mathbb{Z}^{N}$ is defined as:

$$
\Lambda_{n, j}^{m}(\varepsilon):=2^{j} m+\delta_{j}(\varepsilon) n+\Lambda_{j}(\varepsilon):=\left\{2^{j} m+\delta_{j}(\varepsilon) n+l: l \in \Lambda_{j}(\varepsilon)\right\},
$$

where $\Lambda_{j}(\varepsilon)$ is the finite subset of $\mathbb{Z}^{N}$ such that

$$
\Lambda_{j}(\varepsilon):=\left\{0,1, \ldots, \delta_{j}(\varepsilon)\right\}^{N} .
$$

Observe that, (4.20), (4.19) and (4.16) imply that

$$
2^{j \varepsilon N} \leq \zeta_{j, \varepsilon}:=\operatorname{Card}\left(\Lambda_{j}(\varepsilon)\right)=\operatorname{Card}\left(\Lambda_{n, j}^{m}(\varepsilon)\right) \leq 3^{N} 2^{j \varepsilon N}
$$

Also, observe that, for each fixed $j \in \mathbb{N}$ and $m=\left(m_{1}, \ldots, m_{N}\right) \in \mathbb{Z}^{N},(4.20)$, (4.19) and (4.17) entail that

$$
\prod_{v=1}^{N}\left\{2^{j} m_{v}, 2^{j} m_{v}+1, \ldots, 2^{j}\left(m_{v}+1\right)\right\} \subset \bigcup_{n \in \mathcal{S}_{j}} \Lambda_{n, j}^{m}(\varepsilon) .
$$

As a consequence, one has that

$$
\mathbb{Z}^{N}=\bigcup_{m \in \mathbb{Z}^{N}} \bigcup_{n \in \mathcal{S}_{j}} \Lambda_{n, j}^{m}(\varepsilon), \quad \text { for each fixed } j \in \mathbb{N} .
$$

Let us now introduce two important random variables $\mathcal{M}_{n, j}^{m}(\varepsilon)$ and $\mathcal{S}_{n, j}^{m}(\varepsilon)$ associated with each one of the sets of indices $\Lambda_{n, j}^{m}(\varepsilon)$. To this end, one sets

$$
v_{j}:=\left\|g \cdot \widehat{\psi}_{j}\right\|_{L^{2}\left(\mathbb{R}^{N}\right)}^{2}=(2 \pi)^{N}\left\|\mathcal{F}^{-1}(g) * \psi_{j}\right\|_{L^{2}\left(\mathbb{R}^{N}\right)}^{2}
$$


notice that, in view of (3.10), one has

$$
v_{j}=\operatorname{Var}\left(\chi_{j, k}\right)=\mathbb{E}\left(\left|\chi_{j, k}\right|^{2}\right), \quad \text { for all } k \in \mathbb{Z}^{N} .
$$

For all $j \in \mathbb{N}, m \in \mathbb{Z}^{N}$ and $n \in \mathcal{S}_{j}(\varepsilon)$, the random variables $\mathcal{M}_{n, j}^{m}(\varepsilon)$ and $\mathcal{S}_{n, j}^{m}(\varepsilon)$ are defined as:

$$
\mathcal{M}_{n, j}^{m}(\varepsilon):=\sup _{k \in \Lambda_{n, j}^{m}(\varepsilon)}\left|\chi_{j, k}\right|^{2}
$$

and

$$
\mathcal{S}_{n, j}^{m}(\varepsilon):=\zeta_{j, \varepsilon}^{-1} \sum_{k \in \Lambda_{n, j}^{m}(\varepsilon)}\left(\left|\chi_{j, k}\right|^{2}-v_{j}\right)=-v_{j}+\zeta_{j, \varepsilon}^{-1} \sum_{k \in \Lambda_{n, j}^{m}(\varepsilon)}\left|\chi_{j, k}\right|^{2} .
$$

Notice that one can easily derive from (4.27), (4.26) and the two equalities in (4.21) that

$$
\mathcal{S}_{n, j}^{m}(\varepsilon) \leq \mathcal{M}_{n, j}^{m}(\varepsilon)-v_{j}, \quad \text { for all } j \in \mathbb{N}
$$

Remark 4.1 Let $\mathcal{G}$ be the (closed) Gaussian Hilbert space on $\mathbb{R}$ spanned by the centred real-valued Gaussian process $\left\{\chi_{j, k}\right\}_{(j, k) \in \mathbb{N} \times \mathbb{Z}^{N}}$, and let $\overline{\mathcal{P}}_{2}(\mathcal{G})$ be the associated second order chaos as defined in the Definition 2.1 on page 17 in [20]. Then, for all $j \in \mathbb{N}, m \in \mathbb{Z}^{N}$ and $n \in \mathcal{S}_{j}(\varepsilon)$, the random variable $\mathcal{S}_{n, j}^{m}(\varepsilon)$ (see (4.27)) belongs to $\overline{\mathcal{P}}_{2}(\mathcal{G})$. Thus, one knows from Theorem 5.10 on page 62 in [20] that, for any fixed $q \in \mathbb{N}$, there exist two finite and strictly positive universal constants $c^{\prime}(q)$ and $c^{\prime \prime}(q)$, only depending on $q$, such that, for every $(j, m, n)$, one has

$$
c^{\prime}(q)\left(\mathbb{E}\left(\left|\mathcal{S}_{n, j}^{m}(\varepsilon)\right|^{2}\right)\right)^{q} \leq \mathbb{E}\left(\left|\mathcal{S}_{n, j}^{m}(\varepsilon)\right|^{2 q}\right) \leq c^{\prime \prime}(q)\left(\mathbb{E}\left(\left|\mathcal{S}_{n, j}^{m}(\varepsilon)\right|^{2}\right)\right)^{q} .
$$

Our next goal is to provide a suitable upper estimate for $\mathbb{E}\left(\left|\mathcal{S}_{n, j}^{m}(\varepsilon)\right|^{2}\right)$ the second moment of the random variable $\mathcal{S}_{n, j}^{m}(\varepsilon)$. To this end, we need among other things the "continuous and discrete versions" of the Hausdorff-Young inequality which are recalled in the sequel. We mention in passing that these two crucial inequalities can be derived from the Riesz-Thorin interpolation Theorem as explained in the first section of the Chapter 1 in [8].

Lemma 4.2 Let $p \in(1,2]$ be arbitrary and let $p^{\prime} \in[2,+\infty)$ be such that $p^{\prime}=p /(p-1)$ that is $1 / p+1 / p^{\prime}=1$. Assume that $f \in L^{p}\left(\mathbb{R}^{N}\right)$ is an arbitrary function and that 
$\left(f_{n}\right)_{n \in \mathbb{N}}$ is any sequence of functions belonging to $L^{1}\left(\mathbb{R}^{N}\right) \cap L^{p}\left(\mathbb{R}^{N}\right)$ which converges to $f$ in the sense of the $L^{p}\left(\mathbb{R}^{N}\right)$ norm (for instance one can take $f_{n}(x):=f(x) \mathbb{1}_{[-n, n]^{N}}(x)$, for all $n \in \mathbb{N}$ and $\left.x \in \mathbb{R}^{N}\right)$. For each $n \in \mathbb{N}$, the function $\widehat{f}_{n}$ denotes the Fourier transform of $f_{n}$ defined, for all $\xi \in \mathbb{R}^{N}$, in the very usual way: $\widehat{f}_{n}(\xi):=\int_{\mathbb{R}^{N}} e^{-i \xi \cdot x} f_{n}(x) d x$. Then $\widehat{f}_{n}$ belongs to $L^{p^{\prime}}\left(\mathbb{R}^{N}\right)$, moreover the sequence $\left(\widehat{f}_{n}\right)_{n \in \mathbb{N}}$ converges in the sense of the $L^{p^{\prime}}\left(\mathbb{R}^{N}\right)$ norm to a function belonging to $L^{p^{\prime}}\left(\mathbb{R}^{N}\right)$ denoted by $\widehat{f}$ which does not depend on the choice of the sequence $\left(f_{n}\right)_{n \in \mathbb{N}}$ which approximates $f$. The function $\widehat{f}$ is called the Fourier transform of $f$ since it can be identified in the natural way to the Fourier transform of $f$ in the sense of tempered distributions, and it satisfies the "continuous version" of the Hausdorff-Young inequality:

$$
\|\widehat{f}\|_{L^{p^{\prime}\left(\mathbb{R}^{N}\right)}} \leq(2 \pi)^{N / p^{\prime}}\|f\|_{L^{p}\left(\mathbb{R}^{N}\right)}
$$

The following remark explains how the "continuous version" of the Hausdorff-Young inequality is useful for us.

Remark 4.3 One knows from the condition $\left(\mathcal{H}_{2}\right)$ in Theorem 2.7 that there exist $n_{0} \in \mathbb{N}$ and $p_{0} \in[4 / 3,2)$ such that

$$
\mathcal{F}^{-1}\left(g_{\nabla, n_{0}}\right) \in \bigcap_{s \in(0, \bar{s})} B_{p_{0}, \infty}^{s+\nu_{N, p_{0}}}\left(\mathbb{R}^{N}\right)
$$

where

$$
\nu_{N, p_{0}}:=\frac{N\left(2-p_{0}\right)}{2 p_{0}} .
$$

In view of Theorem 2.3 and (2.13), a straightforward consequence of (4.31) is that the function $\mathcal{F}^{-1}(g) * \psi_{j}$ belongs to $L^{p_{0}}\left(\mathbb{R}^{N}\right)$, for all $j \geq n_{0}$. Thus, Lemma 4.2 implies that its Fourier transform $\mathcal{F}\left(\mathcal{F}^{-1}(g) * \psi_{j}\right)=g \cdot \widehat{\psi}_{j}$ belongs to $L^{p_{0}^{\prime}}\left(\mathbb{R}^{N}\right)$ where

$$
p_{0}^{\prime}:=\frac{p_{0}}{p_{0}-1} \in(2,4]
$$

and one has

$$
\left\|g \cdot \widehat{\psi}_{j}\right\|_{L^{p_{0}^{\prime}\left(\mathbb{R}^{N}\right)}} \leq(2 \pi)^{N / p_{0}^{\prime}}\left\|\mathcal{F}^{-1}(g) * \psi_{j}\right\|_{L^{p_{0}\left(\mathbb{R}^{N}\right)}} .
$$

In order to give the statement of the "discrete version" of the Hausdorff-Young inequality, first we need the following definition. 
Definition 4.4 Let $\mathbb{T}^{N}$ be the cube of $\mathbb{R}^{N}$ defined as

$$
\mathbb{T}^{N}:=[0,2 \pi]^{N}
$$

observe that $\mathbb{T}^{N}$ can be viewed as the $N$-dimensional torus, that is the Cartesian product of the unit circle $N$-times with itself. For each fixed $\beta \in[1,+\infty]$, one denotes by $L^{\beta}\left(\mathbb{T}^{N}\right)$ the Banach space over $\mathbb{C}$ consisting in the Lebesgue measurable functions $F$ from $\mathbb{R}^{N}$ to $\mathbb{C}$ which are $(2 \pi \mathbb{Z})^{N}$-periodic (that is, for each $k \in \mathbb{Z}^{N}$ and for Lebesgue almost all $x \in \mathbb{R}^{N}$, one has $\left.F(x+2 \pi k)=F(x)\right)$ and satisfy:

$$
\|F\|_{L^{\beta}\left(\mathbb{T}^{N}\right)}:=\left(\int_{\mathbb{T}^{N}}|F(x)|^{\beta} d x\right)^{1 / \beta}<+\infty .
$$

For any $F \in L^{\beta}\left(\mathbb{T}^{N}\right)$, the sequence of the Fourier coefficients $\mathcal{C}(F)=\left(\mathcal{C}_{r}(F)\right)_{r \in \mathbb{Z}^{N}}$ is defined, for all $r \in \mathbb{Z}^{N}$, as

$$
\mathcal{C}_{r}(F):=\int_{\mathbb{T}^{N}} e^{-i r \cdot x} F(x) d x
$$

It is clear that the sequence $\mathcal{C}(F)$ always belongs to the Banach space $l^{\infty}\left(\mathbb{Z}^{N}\right)$.

Having introduced these notations we are now in a position to give the statement of the "discrete version" of the Hausdorff-Young inequality.

Lemma 4.5 Let $\theta \in(1,2]$ be arbitrary and fixed let $\theta^{\prime} \in[2,+\infty)$ be such that $\theta^{\prime}=$ $\theta /(\theta-1)$ that is one has $1 / \theta+1 / \theta^{\prime}=1$. Then, for any arbitrary function $F \in L^{\theta}\left(\mathbb{T}^{N}\right)$, the sequence of the Fourier coefficients $\mathcal{C}(F)=\left(\mathcal{C}_{r}(F)\right)_{r \in \mathbb{Z}^{N}}$ belongs to the Banach space $l^{\theta^{\prime}}\left(\mathbb{Z}^{N}\right)$ and satisfies the "discrete version" of the Hausdorff-Young inequality:

$$
\|\mathcal{C}(F)\|_{l^{\theta^{\prime}\left(\mathbb{Z}^{N}\right)}}:=\left(\sum_{r \in \mathbb{Z}^{N}}\left|\mathcal{C}_{r}(F)\right|^{\theta^{\prime}}\right)^{1 / \theta^{\prime}} \leq(2 \pi)^{N / \theta^{\prime}}\|F\|_{L^{\theta\left(\mathbb{T}^{N}\right)}} .
$$

The following lemma mainly connects the norm $\left\|g \cdot \widehat{\psi}_{j}\right\|_{L^{p_{0}^{\prime}\left(\mathbb{R}^{N}\right)}}$ in (4.34) to that of a well-chosen function $G_{j}$ in the space $L^{p_{0}^{\prime} / 2}\left(\mathbb{T}^{N}\right)$.

Lemma 4.6 Let $n_{0}$ be as in Remark 4.3. For each integer $j \geq n_{0}$ one denotes by $G_{j}$ the $\mathbb{R}_{+}$-valued $(2 \pi \mathbb{Z})^{N}$-periodic Lebesgue measurable function defined as:

$$
G_{j}(\eta):=\sum_{\kappa \in \mathbb{Z}^{N}}\left|\widehat{\psi}_{0}(\eta+2 \pi \kappa)\right|^{2}\left|g\left(2^{j}(\eta+2 \pi \kappa)\right)\right|^{2}, \quad \text { for all } \eta \in \mathbb{R}^{N}
$$


Observe that this definition makes sense since one knows from (2.7) that the sum in (4.39) only consists in a finite number of terms, for any given $\eta \in \mathbb{R}^{N}$. Let $p_{0} \in[4 / 3,2)$ and $p_{0}^{\prime} \in(2,4]$ be as in Remark 4.3, and let $\theta_{0} \in(1,2]$ be such that

$$
\theta_{0}:=\frac{p_{0}^{\prime}}{2}=\frac{p_{0}}{2\left(p_{0}-1\right)} \text {. }
$$

Then, the function $G_{j}$ belongs to $L^{\theta_{0}}\left(\mathbb{T}^{N}\right)$ and one has

$$
\left\|G_{j}\right\|_{L^{\theta_{0}\left(\mathbb{T}^{N}\right)}} \leq c 2^{-j N / \theta_{0}}\left\|g \cdot \widehat{\psi}_{j}\right\|_{L^{p^{\prime}\left(\mathbb{R}^{N}\right)}}^{2}, \quad \text { for all } j \geq n_{0},
$$

where the constant $c:=2^{N\left(\theta_{0}-1\right) / \theta_{0}}$. Moreover, the sequence of the Fourier coefficients of $G_{j}$, denoted by $\mathrm{C}\left(G_{j}\right)=\left(\mathrm{C}_{r}\left(G_{j}\right)\right)_{r \in \mathbb{Z}^{N}}$, satisfies

$$
\mathcal{C}_{r}\left(G_{j}\right)=\int_{\mathbb{R}^{N}} e^{-i r \cdot \eta}\left|\widehat{\psi}_{0}(\eta)\right|^{2}\left|g\left(2^{j} \eta\right)\right|^{2} d \eta, \quad \text { for all } r \in \mathbb{Z}^{N}
$$

Proof Using (4.39), (4.35), (2.7), the fact that $z \mapsto z^{\theta_{0}}$ is a convex function on $\mathbb{R}_{+}$, the equality $\operatorname{Card}\left(\{0,1\}^{N}\right)=2^{N},(4.40)$, the change of variable $\xi=2^{j} \eta$, and (2.4), one obtains that

$$
\begin{aligned}
\left\|G_{j}\right\|_{L^{\theta_{0}\left(\mathbb{T}^{N}\right)}}^{\theta_{0}} & =\int_{\mathbb{T}^{N}}\left(\sum_{\kappa \in\{0,1\}^{N}}\left|\widehat{\psi}_{0}(\eta-2 \pi \kappa)\right|^{2}\left|g\left(2^{j}(\eta-2 \pi \kappa)\right)\right|^{2}\right)^{\theta_{0}} d \eta \\
& \leq 2^{N\left(\theta_{0}-1\right)} \sum_{\kappa \in\{0,1\}^{N}} \int_{\mathbb{T}^{N}}\left|\widehat{\psi}_{0}(\eta-2 \pi \kappa)\right|^{p_{0}^{\prime}}\left|g\left(2^{j}(\eta-2 \pi \kappa)\right)\right|^{p_{0}^{\prime}} d \eta \\
& =2^{N\left(\theta_{0}-1\right)} \int_{\mathbb{R}^{N}}\left|\widehat{\psi}_{0}(\eta)\right|^{p_{0}^{\prime}}\left|g\left(2^{j} \eta\right)\right|^{p_{0}^{\prime}} d \eta \\
& =2^{N\left(\theta_{0}-1\right)} 2^{-N j} \int_{\mathbb{R}^{N}}\left|\widehat{\psi}_{j}(\xi)\right|^{p_{0}^{\prime}}|g(\xi)|^{p_{0}^{\prime}} d \xi=2^{N\left(\theta_{0}-1\right)} 2^{-N j}\left\|g \cdot \widehat{\psi}_{j}\right\|_{L^{p_{0}^{\prime}\left(\mathbb{R}^{N}\right)}}^{2_{0}},
\end{aligned}
$$

which shows that (4.41) is satified. We mention in passing that (4.41) and the fact that $g \cdot \widehat{\psi}_{j} \in L^{p_{0}^{\prime}}\left(\mathbb{R}^{N}\right)$ (see Remark 4.3) imply that $G_{j} \in L^{\theta_{0}}\left(\mathbb{T}^{N}\right)$. At last, the equality (4.42) easily follows from (4.35), (4.37), (4.39) and the fact $\eta \mapsto e^{-i r \cdot \eta}$ is a $(2 \pi \mathbb{Z})^{N}$-periodic function.

The following remark explains how the "discrete version" of the Hausdorff-Young inequality is useful for us. 
Remark 4.7 Let $\theta_{0} \in(1,2]$ be as in $(4.40)$ and $\theta_{0}^{\prime} \in[2,+\infty)$ such that $1 / \theta_{0}+1 / \theta_{0}^{\prime}=1$, that is

$$
\theta_{0}=\frac{p_{0}}{2\left(p_{0}-1\right)} \quad \text { and } \quad \theta_{0}^{\prime}:=\frac{\theta_{0}}{\theta_{0}-1}=\frac{p_{0}}{2-p_{0}}
$$

where $p_{0} \in[4 / 3,2)$ is as in Remark 4.3. Then, one can derive from Lemmas 4.5 and 4.6 and from Remark 4.3 that, for each $j \geq n_{0}$, the sequence of the Fourier coefficients $\mathcal{C}\left(G_{j}\right)=\left(\mathcal{C}_{r}\left(G_{j}\right)\right)_{r \in \mathbb{Z}^{N}}$ belongs to the Banach space $l^{\theta_{0}^{\prime}}\left(\mathbb{Z}^{N}\right)$ and satisfies

$$
\left\|\mathcal{C}\left(G_{j}\right)\right\|_{l^{\theta_{0}^{\prime}\left(\mathbb{Z}^{N}\right)}} \leq c 2^{-j N / \theta_{0}}\left\|\mathcal{F}^{-1}(g) * \psi_{j}\right\|_{L^{p_{0}\left(\mathbb{R}^{N}\right)}}^{2},
$$

where $c>0$ is a finite constant not depending on $j$.

The following lemma provides a suitable upper estimate for $\mathbb{E}\left(\left|\mathcal{S}_{n, j}^{m}(\varepsilon)\right|^{2}\right)$.

Lemma 4.8 Let $n_{0} \in \mathbb{N}, p_{0} \in[3 / 4,2)$ and $\nu_{N, p_{0}}$ be as in Remark 4.3. Let $\theta_{0}^{\prime} \in[2,+\infty)$ be as in (4.43). There exists a finite constant c such that, for all integer $j \geq n_{0}, m \in \mathbb{Z}^{N}$ and $n \in \mathcal{S}_{j}(\varepsilon)$, one has

$$
\mathbb{E}\left(\left|\mathcal{S}_{n, j}^{m}(\varepsilon)\right|^{2}\right) \leq c \zeta_{j, \varepsilon}^{-2 / \theta_{0}^{\prime}}\left(2^{j \nu_{N, p_{0}}}\left\|\mathcal{F}^{-1}(g) * \psi_{j}\right\|_{L^{p_{0}\left(\mathbb{R}^{N}\right)}}\right)^{4}
$$

where $\zeta_{j, \varepsilon}$ and $\mathcal{S}_{n, j}^{m}(\varepsilon)$ are as in (4.21) and (4.27).

Proof One denotes by $c_{1}$ the constant $c$ in (4.44), and one sets $c_{2}:=2 c_{1}^{2}$. Using the first equality in (4.27), (4.25), the classical equality (see e.g. Theorem 3.9 on page 26 in $[20])$

$$
\operatorname{Cov}\left(\left|Z_{1}\right|^{2},\left|Z_{2}\right|^{2}\right)=2\left(\mathbb{E}\left(Z_{1} Z_{2}\right)\right)^{2}
$$

which holds for any $\mathbb{R}^{2}$-valued centred Gaussian vector $\left(Z_{1}, Z_{2}\right)$, (3.9), the isometry property (1.5) of the stochastic integral $\int_{\mathbb{R}^{N}}(\cdot) d \widehat{W},(4.19),(2.4)$, the change of variable $\eta=2^{-j} \xi$, (4.42), Hölder inequality, the fact that $\zeta_{j, \varepsilon}:=\operatorname{Card}\left(\Lambda_{j}(\varepsilon)\right)$, the definition of the norm $\|\cdot\|_{l^{\theta_{0}^{\prime}\left(\mathbb{Z}^{N}\right)}},(4.44),(4.43)$, and (4.32), one gets, for all integer $j \geq n_{0}, m \in \mathbb{Z}^{N}$ 
and $n \in \mathcal{S}_{j}(\varepsilon)$, that

$$
\begin{aligned}
& \mathbb{E}\left(\left|\mathcal{S}_{n, j}^{m}(\varepsilon)\right|^{2}\right)=\zeta_{j, \varepsilon}^{-2} \sum_{k^{\prime}, k^{\prime \prime} \in \Lambda_{n, j}^{m}(\varepsilon)} \operatorname{Cov}\left(\left|\chi_{j, k^{\prime}}\right|^{2},\left|\chi_{j, k^{\prime \prime}}\right|^{2}\right) \\
& =2 \zeta_{j, \varepsilon}^{-2} \sum_{k^{\prime}, k^{\prime \prime} \in \Lambda_{n, j}^{m}(\varepsilon)}\left(\mathbb{E}\left(\chi_{j, k^{\prime}} \chi_{j, k^{\prime \prime}}\right)\right)^{2} \\
& =2 \zeta_{j, \varepsilon}^{-2} \sum_{k^{\prime}, k^{\prime \prime} \in \Lambda_{n, j}^{m}(\varepsilon)}\left(\int_{\mathbb{R}^{N}} e^{i 2^{-j}\left(k^{\prime \prime}-k^{\prime}\right) \cdot \xi}\left|\widehat{\psi}_{j}(\xi)\right|^{2}|g(\xi)|^{2} d \xi\right)^{2} \\
& =2^{2 j N+1} \zeta_{j, \varepsilon}^{-2} \sum_{l^{\prime}, l^{\prime \prime} \in \Lambda_{j}(\varepsilon)}\left(\int_{\mathbb{R}^{N}} e^{i\left(l^{\prime \prime}-l^{\prime}\right) \cdot \eta}\left|\widehat{\psi}_{0}(\eta)\right|^{2}\left|g\left(2^{j} \eta\right)\right|^{2} d \eta\right)^{2} \\
& =2^{2 j N+1} \zeta_{j, \varepsilon}^{-1} \sum_{l^{\prime} \in \Lambda_{j}(\varepsilon)}\left(\left(\zeta_{j, \varepsilon}^{-1} \sum_{l^{\prime \prime} \in \Lambda_{j}(\varepsilon)}\left|\mathcal{C}_{l^{\prime}-l^{\prime \prime}}\left(G_{j}\right)\right|^{2}\right)^{1 / 2}\right)^{2} \\
& \leq 2^{2 j N+1} \zeta_{j, \varepsilon}^{-1} \sum_{l^{\prime} \in \Lambda_{j}(\varepsilon)}\left(\left(\zeta_{j, \varepsilon}^{-1} \sum_{l^{\prime \prime} \in \Lambda_{j}(\varepsilon)}\left|\varrho_{l^{\prime}-l^{\prime \prime}}\left(G_{j}\right)\right|^{\theta_{0}^{\prime}}\right)^{1 / \theta_{0}^{\prime}}\right)^{2} \\
& \leq 2^{2 j N+1} \zeta_{j, \varepsilon}^{-2 / \theta_{0}^{\prime}}\left\|\mathcal{C}\left(G_{j}\right)\right\|_{l^{\theta_{0}^{\prime}\left(\mathbb{Z}^{N}\right)}}^{2} \\
& \leq 2^{2 j N+1} \zeta_{j, \varepsilon}^{-2 / \theta_{0}^{\prime}}\left(c_{1} 2^{-j N / \theta_{0}}\left\|\mathcal{F}^{-1}(g) * \psi_{j}\right\|_{L^{p_{0}\left(\mathbb{R}^{N}\right)}}^{2}\right)^{2} \\
& \leq c_{2} \zeta_{j, \varepsilon}^{-2 / \theta_{0}^{\prime}}\left(2^{j \nu_{N, p_{0}}}\left\|\mathcal{F}^{-1}(g) * \psi_{j}\right\|_{L^{p_{0}\left(\mathbb{R}^{N}\right)}}\right)^{4},
\end{aligned}
$$

which shows that (4.45) is satisfied.

We are now in a position to prove Theorem 2.7.

Proof of Theorem 2.7 Throughout the proof, the arbitrarily small fixed (strictly) positive real number $\varepsilon$ is the same as in (4.16). Moreover, $n_{0} \in \mathbb{N}, p_{0} \in[3 / 4,2)$ and $\nu_{N, p_{0}}$ are assumed to be as in Remark 4.3, and $\theta_{0}^{\prime} \in[2,+\infty)$ is assumed to be as in (4.43). One knows from (1.10) and Theorem 2.3 that there exist an infinite subset of distinct integers $\mathbb{J}(\varepsilon) \subseteq \mathbb{N} \cap\left[n_{0},+\infty\right)$ and a (strictly) positive constant $c_{1}$ such that

$$
\left\|\mathcal{F}^{-1}(g) * \psi_{j}\right\|_{L^{2}\left(\mathbb{R}^{N}\right)} \geq c_{1} 2^{-j \bar{s}-j\left(N \varepsilon / 8 \theta_{0}^{\prime}\right)}, \quad \text { for all } j \in \mathbb{J}(\varepsilon)
$$

On the other hand, (4.31), Theorem 2.3 and (2.13) imply that there is a finite positive constant $c_{2}$ such that

$$
2^{j \nu_{N, p_{0}}}\left\|\mathcal{F}^{-1}(g) * \psi_{j}\right\|_{L^{p_{0}\left(\mathbb{R}^{N}\right)}} \leq c_{2} 2^{-j \bar{s}+j\left(N \varepsilon / 8 \theta_{0}^{\prime}\right)}, \quad \text { for all } j \geq n_{0}
$$


Combining (4.46) and (4.47), it follows that, for some finite positive constant $c_{3}$, one has

$$
\left(\frac{2^{j \nu_{N, p_{0}}}\left\|\mathcal{F}^{-1}(g) * \psi_{j}\right\|_{L^{p_{0}\left(\mathbb{R}^{N}\right)}}}{\left\|\mathcal{F}^{-1}(g) * \psi_{j}\right\|_{L^{2}\left(\mathbb{R}^{N}\right)}}\right)^{4} \leq c_{3} 2^{j\left(N \varepsilon / \theta_{0}^{\prime}\right)}, \quad \text { for all } j \in \mathbb{J}(\varepsilon) .
$$

Next, for all $j \in \mathbb{J}(\varepsilon), m \in \mathbb{Z}^{N}$ and $n \in \mathcal{S}_{j}(\varepsilon)$, one denotes by $D_{n, j}^{m}(\varepsilon)$ the event defined as:

$$
D_{n, j}^{m}(\varepsilon):=\left\{\mathcal{M}_{n, j}^{m}(\varepsilon) \leq 2^{-1} v_{j}\right\}
$$

where the random variable $\mathcal{M}_{n, j}^{m}(\varepsilon)$ and the variance $v_{j}$ are as in (4.26) and (4.25) (see also (4.24)). Next, let $q \in \mathbb{N}$ be a fixed arbitrarily large integer. Using (4.49), (4.28), the Markov inequality, and the second inequality in (4.29), one obtains, for all $j \in \mathbb{J}(\varepsilon)$, that

$$
\begin{aligned}
\mathbb{P}\left(D_{n, j}^{m}(\varepsilon)\right) & \leq \mathbb{P}\left(\mathcal{S}_{n, j}^{m}(\varepsilon) \leq-2^{-1} v_{j}\right) \\
& \leq \mathbb{P}\left(\left|\mathcal{S}_{n, j}^{m}(\varepsilon)\right| \geq 2^{-1} v_{j}\right) \\
& \leq 4^{q} v_{j}^{-2 q} \mathbb{E}\left(\left|\mathcal{S}_{n, j}^{m}(\varepsilon)\right|^{2 q}\right) \leq c_{4}(q)\left(v_{j}^{-2} \mathbb{E}\left(\left|\mathcal{S}_{n, j}^{m}(\varepsilon)\right|^{2}\right)\right)^{q}
\end{aligned}
$$

where $c_{4}(q)$ is a positive finite constant only depending on $q$. Next putting together (4.45), (4.24), (4.48), (4.50) and the first inequality in (4.21), it follows that, for some positive finite constant $c_{5}$ not depending on $(j, m, n)$, one has

$$
\mathbb{P}\left(D_{n, j}^{m}(\varepsilon)\right) \leq c_{5} 2^{-j N\left(q \varepsilon / \theta_{0}^{\prime}\right)}, \quad \text { for all } j \in \mathbb{J}(\varepsilon), m \in \mathbb{Z}^{N} \text { and } n \in \mathcal{S}_{j}(\varepsilon) \text {. }
$$

Then, one can derive from the second inequality in (4.18) that, for some positive finite constant $c_{6}$ not depending on $(j, m)$, one has

$$
\begin{aligned}
\mathbb{P}\left(\bigcup_{n \in \mathcal{S}_{j}(\varepsilon)} D_{n, j}^{m}(\varepsilon)\right) & \leq \sum_{n \in \mathcal{S}_{j}(\varepsilon)} \mathbb{P}\left(D_{n, j}^{m}(\varepsilon)\right) \\
& \leq c_{6} 2^{-j N\left(\left(q \varepsilon / \theta_{0}^{\prime}\right)-(1-\varepsilon)\right)}, \quad \text { for all }(j, m) \in \mathbb{J}(\varepsilon) \times \mathbb{Z}^{N}
\end{aligned}
$$

Since $q \in \mathbb{N}$ is arbitrary, it can be chosen so that $\left(q \varepsilon / \theta_{0}^{\prime}\right)-(1-\varepsilon)>0$, then it results from (4.51) that

$$
\sum_{j \in \mathbb{J}(\varepsilon)} \mathbb{P}\left(\bigcup_{n \in \mathcal{S}_{j}(\varepsilon)} D_{n, j}^{m}(\varepsilon)\right)<+\infty, \quad \text { for all } m \in \mathbb{Z}^{N}
$$


Thus, denoting by $\bar{D}_{n, j}^{m}(\varepsilon)$ the complement of the event $D_{n, j}^{m}(\varepsilon)$ defined in (4.49), that is

$$
\bar{D}_{n, j}^{m}(\varepsilon):=\left\{\mathcal{M}_{n, j}^{m}(\varepsilon)>2^{-1} v_{j}\right\}
$$

it results from the Borel-Cantelli Lemma that

$$
\mathbb{P}\left(\bigcup_{J \in \mathbb{J}(\varepsilon)} \bigcap_{j \geq J, j \in \mathbb{J}(\varepsilon)} \bigcap_{n \in \mathcal{S}_{j}(\varepsilon)} \bar{D}_{n, j}^{m}(\varepsilon)\right)=1, \quad \text { for all } m \in \mathbb{Z}^{N} .
$$

Since $\mathbb{Z}^{N}$ is a countable set, (4.53) implies that the probability of the event

$$
\Omega_{4}^{*}(\varepsilon):=\bigcap_{m \in \mathbb{Z}^{N}} \bigcup_{J \in \mathbb{J}(\varepsilon)} \bigcap_{j \geq J, j \in \mathbb{J}(\varepsilon)} \bigcap_{n \in \mathcal{S}_{j}(\varepsilon)} \bar{D}_{n, j}^{m}(\varepsilon)
$$

is equal to 1 . Next, let $\omega \in \Omega_{4}^{*}(\varepsilon)$ be arbitrary and fixed, it results from (4.54) and (4.52) that:

$$
\begin{aligned}
\forall m \in \mathbb{Z}^{N}, \exists \widetilde{J}(m, \omega) \in \mathbb{J}(\varepsilon) \text { s.t. } \forall j \in \mathbb{J}(\varepsilon),(j \geq \widetilde{J}(m, \omega)) \\
\Longrightarrow\left(\forall n \in \mathcal{S}_{j}(\varepsilon), \mathcal{M}_{n, j}^{m}(\varepsilon, \omega)>2^{-1} v_{j}\right) .
\end{aligned}
$$

On the other hand, observe that, using the same notations as in Part (ii) of Remark 3.4, for each $\tau \in \mathbb{R}^{N}$ and for every $j \in \mathbb{N}$, one has

$$
k_{j}(\tau)-2^{j} k_{0}(\tau) \in\left\{0, \ldots, 2^{j}-1\right\}^{N} .
$$

Then, it follows from (4.16), (4.17) and (4.20), that there exist $n_{j}(\tau) \in \mathcal{S}_{j}(\varepsilon)$ and $l_{j}(\tau) \in \Lambda_{j}(\varepsilon)$ such that

$$
k_{j}(\tau)-2^{j} k_{0}(\tau)=\delta_{j}(\varepsilon) n_{j}(\tau)+l_{j}(\tau)
$$

Thus, one knows from (4.56) and (4.19) that

$$
k_{j}(\tau) \in \Lambda_{n_{j}(\tau), j}^{k_{0}(\tau)}(\varepsilon), \quad \text { for all } j \in \mathbb{N} .
$$

Moreover, it results from (4.57), (4.19), the inequality $\left|2^{-j} k_{j}(\tau)-\tau\right| \leq 2^{-j} \sqrt{N}$ (see (3.22)), the triangle inequality, and (4.16) that

$$
\max \left\{\left|2^{-j} k-\tau\right|: k \in \Lambda_{n_{j}(\tau), j}^{k_{0}(\tau)}\right\} \leq c_{7} 2^{-j(1-\varepsilon)}, \quad \text { for all } j \in \mathbb{N}
$$


where the constant $c_{7}:=3 \sqrt{N}$. Therefore, setting $c_{8}:=c_{7}+\sqrt{N}=4 \sqrt{N}$ one has

$$
\prod_{u=1}^{N}\left[2^{-j} k_{u}, 2^{-j}\left(k_{u}+1\right)\right] \subset \mathcal{B}\left(\tau, c_{8} 2^{-j(1-\varepsilon)}\right), \quad \text { for all } j \in \mathbb{N} \text { and } k \in \Lambda_{n_{j}(\tau), j}^{k_{0}(\tau)}(\varepsilon),
$$

where the integers $k_{1}, \ldots, k_{N}$ are the coordinates of $k$. Thus, combining (4.26), (3.23) and (4.58), one obtains that

$$
\mathcal{M}_{\chi}\left(\tau, c_{8} 2^{-j(1-\varepsilon)}\right) \geq \sqrt{\mathcal{M}_{n_{j}(\tau), j}^{k_{0}(\tau)}(\varepsilon)}, \quad \text { for all }(j, \tau) \in \mathbb{N} \times \mathbb{R}^{N} .
$$

Next, observe that one knows from (4.55) with $m=k_{0}(\tau)$ and $n=n_{j}(\tau),(4.24),(4.46)$ and the inequality $\theta_{0}^{\prime} \geq 2$, that

$$
\limsup _{j \rightarrow+\infty}\left\{2^{j(\bar{s}+N \varepsilon)} \sqrt{\mathcal{M}_{n_{j}(\tau), j}^{k_{0}(\tau)}(\varepsilon, \omega)}\right\}=+\infty, \quad \text { for all }(\tau, \omega) \in \mathbb{R}^{N} \times \Omega_{4}^{*}(\varepsilon) .
$$

Therefore (4.59) implies that

$$
\limsup _{j \rightarrow+\infty}\left\{2^{j(\bar{s}+N \varepsilon)} \mathcal{M}_{\chi}\left(\tau, c_{8} 2^{-j(1-\varepsilon)}, \omega\right)\right\}=+\infty, \quad \text { for all }(\tau, \omega) \in \mathbb{R}^{N} \times \Omega_{4}^{*}(\varepsilon),
$$

and consequently that

$$
\limsup _{r>0, r \rightarrow 0}\left\{r^{-(1+\varepsilon)(\bar{s}+N \varepsilon) /(1-\varepsilon)} \mathcal{M}_{X}\left(\tau, r^{(1+\varepsilon)}, \omega\right)\right\}=+\infty, \quad \text { for all }(\tau, \omega) \in \mathbb{R}^{N} \times \Omega_{4}^{*}(\varepsilon) \text {. }
$$

Next, notice that since $\bar{s} \in(0,1)$, there exists $\varepsilon_{0}>0$ which only depends on $\bar{s}$ and $N$, such that one has $\bar{s}<(1+\varepsilon)(\bar{s}+N \varepsilon) /(1-\varepsilon)<1$, for all $\varepsilon \in\left(0, \varepsilon_{0}\right)$. In view of the fact that $\varepsilon$ is arbitrarily small, one can assume without any restriction that it belongs to the interval $\left(0, \varepsilon_{0}\right)$. Then, $(3.24)$ with $\alpha=(1+\varepsilon)(\bar{s}+N \varepsilon) /(1-\varepsilon)$ and $\gamma=1+\varepsilon$, and (4.60) entail that

$$
\limsup _{r>0, r \rightarrow 0}\left\{r^{-(1+\varepsilon)(\bar{s}+N \varepsilon) /(1-\varepsilon)} \operatorname{Osc}_{X}(\tau, r, \omega)\right\}=+\infty, \quad \text { for all }(\tau, \omega) \in \mathbb{R}^{N} \times \Omega_{5}^{*}(\varepsilon),
$$

where $\Omega_{5}^{*}(\varepsilon)$ is the event of probability 1 , not depending on $\tau$, defined as $\Omega_{5}^{*}(\varepsilon):=$ $\Omega_{4}^{*}(\varepsilon) \cap \Omega_{1}^{*}$. Next, combining (1.13) and (4.61) one obtains that

$$
\alpha_{X}(\tau, \omega) \leq \frac{(1+\varepsilon)(\bar{s}+N \varepsilon)}{(1-\varepsilon)}, \quad \text { for all }(\tau, \omega) \in \mathbb{R}^{N} \times \Omega_{5}^{*}(\varepsilon) .
$$


Next, one denotes by $\Omega_{5}^{*}$ the event of probability 1 , not depending on $\tau$, defined as:

$$
\Omega_{5}^{*}:=\bigcap_{\varepsilon \in \mathbb{Q} \cap\left(0, \varepsilon_{0}\right)} \Omega_{5}^{*}(\varepsilon),
$$

where $\mathbb{Q}$ is the countable set of the rational numbers. Then, in view of (4.62), using the equality

$$
\lim _{\varepsilon>0, \varepsilon \rightarrow 0} \frac{(1+\varepsilon)(\bar{s}+N \varepsilon)}{(1-\varepsilon)}=\bar{s}
$$

one gets that

$$
\alpha_{X}(\tau, \omega) \leq \bar{s} \quad \text { for all }(\tau, \omega) \in \mathbb{R}^{N} \times \Omega_{5}^{*} .
$$

Finally, combining (4.63) and Remark 1.3, it turns out that (1.16) holds when $\Omega^{*}:=\Omega_{5}^{*}$.

\section{Acknowledgements}

This work has been partially supported by the Labex CEMPI (ANR-11-LABX-000701) and the GDR 3475 (Analyse Multifractale et Autosimilarité).

\section{References}

[1] R. J. Adler (1990), An introduction to continuity, extrema, and related topics for general Gaussian processes. Lecture Notes Monograph Series, Volume 12.

[2] P. Andersson (1997), Characterization of pointwise Hölder regularity. Appl. Comp. Harmon. Anal. 4, 429-443.

[3] A. Ayache (2019), Multifractional stochastic fields: wavelet strategies in multifractional frameworks. World Scientific.

[4] A. Ayache (2013), Continuous Gaussian multifractional processes with random pointwise Hölder regularity. J. Theoret. Probab. 26, 72-93.

[5] A. Ayache and J. Hamonier (2014), Linear multifractional stable motion: fine path properties. Rev. Mat. Iberoamericana 30, 1301-1354.

[6] P. Balança (2015), Some sample path properties of multifractional Brownian motion. Stoch. Processes Appl. 125, 3823-3850. 
[7] P. Balança (2014), Fine regularity of Lévy processes and linear (multi)fractional stable motion. Electron. J. Probab. 19, 1-37.

[8] J. Bergh and J. Löfström (1976), Interpolation spaces an introduction. Springer-Verlag.

[9] S.M. Berman (1972), Gaussian sample function: uniform dimension and Hölder conditions nowhere. Nagoya Math. J. 46, 63-86.

[10] S.M. Berman (1973), Local nondeterminism and local times of Gaussian processes. Indiana Univ. Math. J. 23, 69-94.

[11] J. Bertoin and S. Jaffard (1997), Solutions multifractales de l'équation de burgers. Matapli 52, 19-28.

[12] H. Cramer and M.R. Leadbetter (1967), Stationary and related stochastic processes. Wiley \& Sons.

[13] P. Embrechts and M. Maejima (2002), Selfsimilar processes. Princeton University Press.

[14] K. Falconer (1990), Fractal geometry - mathematical foundations and applications. Wiley \& Sons.

[15] A. Fraysse (2010), Regularity criteria of almost every function in a Sobolev space. $J$. Func. Anal. 258, 1806-1821.

[16] A. Fraysse and S. Jaffard (2006), How smooth is almost every function in a Sobolev space? Rev. Mat. Iberoamericana 22, 663-682.

[17] S. Jaffard (2004), Wavelet techniques in multifractal analysis, Fractal Geometry and Applications: A Jubilee of Benoît Mandelbrot, M. Lapidus and M. van Frankenhuijsen Eds., Proceedings of Symposia in Pure Mathematics, AMS, Vol. 72, Part 2, 91-152.

[18] S. Jaffard (1999), The multifractal nature of Lévy processes. Probab. Th. Rel. Fields 114, 207-227.

[19] S. Jaffard, Y. Meyer and R. Ryan (2001), Wavelets: tools for science and technology. SIAM, Philadelphia, PA.

[20] S. Janson (1997), Gaussian Hilbert spaces. Cambridge University Press.

[21] M. Ledoux and M. Talagrand (1991), Probability in Banach spaces. Springer-Verlag, Berlin. 
[22] L.D. Pitt (1978), Local times for Gaussian vector fields. Indiana Univ. Math. J. 27, 309-330.

[23] G. Samorodnitsky and M.S. Taqqu (1994), Stable non-Gaussian random processes: stochastic models with infinite variance. Chapman \& Hall, New York.

[24] S. Seuret (2021), A survey on prescription of multifractal behavior, Fractal Geometry and Stochastics VI, U. Freiberg, B. Hambly, M. Hinz and S. Winter Eds., Birkhäuser, $47-70$.

[25] E.M. Stein and G. Weiss (1971), Introduction to Fourier analysis on Euclidean spaces. Princeton University Press.

[26] Y. Xiao (2006), Properties of local nondeterminism of Gaussian and stable random fields and their applications. Ann. Fac. Sci. Toulouse Math. XV, 157-193.

[27] Y. Xiao (1997), Hölder conditions for the local times and the Hausdorff measure of the level sets of Gaussian random fields. Probab. Th. Rel. Fields 109, 129-157.

[28] A.M. Yaglom (1997), Correlation theory of stationary and related random functions(I). Springer-Verlag.

Antoine Ayache: Univ. Lille,

CNRS, UMR 8524 - Laboratoire Paul Painlevé,

F-59000 Lille, France

E-mail: antoine.ayache@univ-lille.fr 